\title{
LED lighting affects plant growth, morphogenesis and phytochemical contents of Myrtus communis L. in vitro
}

\author{
Monika Cioć $^{1} \cdot$ Agnieszka Szewczyk $^{2} \cdot$ Marek Żupnik $^{3} \cdot$ Andrzej Kalisz $^{4}$. \\ Bożena Pawłowska ${ }^{1}$
}

Received: 21 July 2017 / Accepted: 15 October 2017 / Published online: 9 November 2017

(C) The Author(s) 2017. This article is an open access publication

\begin{abstract}
The influence of light quality and cytokinin content in media on growth, development, photosynthetic pigments and secondary metabolite content of Myrtus communis $\mathrm{L}$. was evaluated in an in vitro culture. Various treatments with light emitting diodes (LEDs): $100 \%$ blue (B), a mix of $70 \%$ red and $30 \%$ blue (RB) and $100 \%$ red were applied and compared with a traditional fluorescent lamp as control. Axillary shoots were incubated on Murashige and Skoog medium with $30 \mathrm{~g} \mathrm{dm}^{-3}$ sucrose, $0.5 \%$ BioAgar, $0.5 \mu \mathrm{M}$ 1-naphthaleneacetic acid and different concentrations of 6-benzyladenine (BA): 1, 2.5 and $5 \mu \mathrm{M}$. Cultures were maintained for 6 weeks in $23 / 21 \pm 1{ }^{\circ} \mathrm{C}$ (day/night), $80 \%$ relative humidity and $16 / 8 \mathrm{~h}$ photoperiod; photosynthetic photon flux density (PPFD) was $35 \mu \mathrm{mol} \mathrm{m} \mathrm{m}^{-2} \mathrm{~s}^{-1}$ in all treatments. Light spectra and BA content in media affected biometrical and phytochemical M. communis properties. Red LEDs and $5 \mu \mathrm{M}$ BA resulted in the highest multiplication rate. The highest shoots were obtained under red LEDs, but with the lowest concentration of cytokinin in media. Fresh weight was greatest on LEDs containing blue light in the spectrum (B and RB); moreover, $5 \mu \mathrm{M}$ BA increased dry
\end{abstract}

Communicated by Sergio J. Ochatt.

Monika Cioć

m.cioc@ogr.ur.krakow.pl

1 Department of Ornamental Plants, University of Agriculture in Krakow, 29 Listopada 54 Avenue, 31-425 Kraków, Poland

2 Department of Pharmaceutical Botany, Jagiellonian University Medical College, Medyczna 9 Street, 30-688 Kraków, Poland

3 PXM, Podłęże 654, 32-003 Podłęże, Poland

4 Department of Vegetable and Medicinal Plants, University of Agriculture in Krakow, 29 Listopada 54 Avenue, 31-425 Kraków, Poland weight. Photosynthetic pigment levels were lower under LED light compared to control lamps. Phenolic acids and flavonoids were identified in $M$. communis leaf extracts. Myricetin was the major constituent with highest concentration under red LEDs and highest BA level.

Keywords Myrtle $\cdot$ Light quality $\cdot$ Photosynthetic pigments $\cdot$ Secondary metabolites $\cdot$ HPLC
Abbreviations
LED Light emitting diode
B $\quad 100 \%$ blue LED
RB $\quad 70 \%$ red and $30 \%$ blue LED
$\mathrm{R} \quad 100 \%$ red LED
C Control fluorescent light
BA 6-Benzyladenine
NAA 1-Naphthaleneacetic acid
MS Murashige and Skoog medium
HPLC High performance liquid chromatography
PPFD Photosynthetic photon flux density

\section{Introduction}

In recent years, there has been increasing interest in the use of medicinal plants as a source of healthy raw materials (Scarpa et al. 2000). According to Touaibia and Chaouch (2015), more than $25 \%$ of medicines is directly or indirectly derived from plants. This is related to the preventive action of antioxidants against "civilisation diseases", including cancer (Aidi Wannes et al. 2010; Pereira et al. 2012; Goncalves et al. 2013; Bouaziz et al. 2015), and related to the abuse of synthetic drugs, the discovery of adverse side effects and high cost of conventional medicinal products (Aleksic and Knezevic 2014). 
Myrtus communis L., also known as true myrtle, is a perennial, evergreen shrub or small tree, typical of the Mediterranean region (Nassar et al. 2010; Sumbul et al. 2011; Aleksic and Knezevic 2014; Alipour et al. 2014; Asgarpanah and Ariamanesh 2015; Yildirim et al. 2015). It is the only species of the Myrtus genus that occurs in this region (Canhoto et al. 1999). The species is very important for the forestation of costal zones damaged by fires. Moreover, in Europe, it is widely known and used as an ornamental plant in the florist and garden industry (Nobre 1997; Ruffoni et al. 2010), and as a potted plant (Jędrzejko et al. 1997). For many years, it has been propagated by seeds or woody stem cuttings (Ruffoni et al. 2010; Lim 2012), and is currently also produced in vitro (Ruffoni et al. 2010). Different parts of M. communis (leaves, flowers, fruits) contain many components significant for medicine, food, liqueur and cosmetic industries, thus the production of this plant in large numbers is greatly needed (Amensour et al. 2009; Aidi Wannes et al. 2010; Romani et al. 2012).

Myrtus communis has been used in medical practice for many years and it exhibits therapeutic effects (Romani et al. 2004; Kalachanis and Psaras 2005; Gardeli et al. 2008; Yoshimura et al. 2008; Frohne and Classen 2006; Goncalves et al. 2013; Taheri et al. 2013; Aleksic and Knezevic 2014; Alipour et al. 2014; Asgarpanah and Ariamanesh 2015; Bouaziz et al. 2015; Yildirim et al. 2015). Healing properties are related to the content of volatile oils, but also phenolic compounds, which can be divided into phenolic acids, flavonoids and tannins that have a strong antioxidant effect (Romani et al. 1999, 2012; Balasundram et al. 2006; Gardeli et al. 2008; Yoshimura et al. 2008; Pereira et al. 2012; Aleksic and Knezevic 2014). Currently, vademecums of medicinal plants provide possible $M$. communis applications and recommendations in the form of ready-made medicines (Hoppe 1975; Frohne and Classen 2006).

Polyphenols are one of the most important secondary metabolites found in M. communis L. leaf extracts (Tumen et al. 2012). They have been shown to protect the metabolism of cells exposed to high temperatures and excess UV-B radiation (Romani et al. 1999). Moreover, these compounds may reduce the risk of some chronic diseases at higher dietary intakes (Romani et al. 2004). According to Hayder et al. (2008), there are approximately 4000 known structures of polyphenol compounds. They have a number of biological properties, including antioxidant, antimicrobial (Mansouri et al. 2001; Amensour et al. 2009), antitumor and antimutagenic properties (Hayder et al. 2008). Plant phenolics are biosynthesised by two basic pathways: the shikimic acid pathway and the malonic acid pathway, the former of which is responsible for the synthesis of most phenolic compounds in plants (Lattanzio 2013). They have the capacity to neutralise free radicals and reduce their harmful effects on the human body (Aidi Wannes et al. 2010; Goncalves et al.
2013; Aleksic and Knezevic 2014; Asgarpanah and Ariamanesh 2015; Bouaziz et al. 2015). Their antioxidant activity depends on the number and position of phenolic hydroxyls in aromatic ring moieties (Aleksic and Knezevic 2014). The main compounds responsible for the flavor and scent of M. communis oil are monoterpenes: 1,8-cineole, myrtenyl acetate, $\alpha$-pinene, myrtenol, and limonene (Gardeli et al. 2008). Among the flavonoids, myricetin, quercetin, catechin, and their derivatives, have so far been found in M. communis leaves and stems (Aleksic and Knezevic 2014; Asgarpanah and Ariamanesh 2015). Myricetin is a substance specific to the family Myrtaceae (Haron et al. 1992), exhibiting antibacterial, antiviral, antioxidant, anti-inflammatory, antiallergic, anticoagulant, antitumor and antimicrobial properties (Aleksic and Knezevic 2014), and studies have shown that it is a much stronger antioxidant than traditional vitamins (Miean and Mohamed 2001). Other researchers reported that myricetin can also be detected in red grape wines (Vitrac et al. 2004), similar to catechin, which is known to be the most abundant monomeric flavon-3-ol in wines. Alamanni and Cossu (2004) confirmed that the antioxidant activity of liqueurs obtained from M. communis leaves was comparable to red wines. It has also been shown that $M$. communis can, due to the presence of these specific substances, have therapeutic effects in the treatment of some diseases (Benkhayal et al. 2009; Ahmadvand and Bagheri 2011; Sumbul et al. 2011; Goncalves et al. 2013; Bouaziz et al. 2015). Moreover, M. communis leaves extracts are practically non-toxic, very safe, and exerted significant therapeutic effects compared to a control group of medinices (Nassar et al. 2010).

Meanwhile, despite numerous advantages and beneficial properties, environmental pollution, heterogeneity of wild plant material and its inaccessibility in some areas make the collected herbal material not only slightly toxic, but also the quality of raw material would not be homogenous (Magherini 1988; Scarpa et al. 2000). There is potential for the use of in vitro plant cultures for obtaining medicinal products from plants propagated in this manner. In this way, genetically homogeneous and healthy material is obtained, and is also produced in large quantities in a short period of time (Pierik 1987; Scarpa et al. 2000). This creates new opportunities for the commercial use of the $M$. communis on a larger scale. Despite many advantages it is still not very popular in economic use because of the low yield (Gardeli et al. 2008). Although there are many difficulties with respect to in vitro cultures of woody plants compared to herbaceous plants, currently $M$. communis is considered to be a model plant for woody shrub tissue cultures (Mascarello et al. 2009; Parra et al. 2001; Ruffoni et al. 2010). The protocols are known and there are reports of in vitro $M$. communis propagation and proliferation (Nobre 1997; Parra and Amo-Marco 1998; Ruffoni et al. 2010). New procedures are investigated and examined also for commercial use (Rezaee and Kamali 
2014). Şan et al. (2015) reports the application of thidiazuron, 6-benzylaminopurine and naphthalene acetic acid for shoot proliferation and rooting of $M$. communis clone. Aka Kaçar et al. (2017) tested influence of activated charcoal and indole-3-butyric acid on the rooting stage. The use of in vitro techniques allows the rapid multiplication of disease-free and true-to-type selected clones (Nobre 1997; Rezaee and Kamali 2014) of $M$. communis, which could be essential in obtaining plants with sufficient quantities of beneficial compounds. However, to our knowledge, no studies have reported the composition or biological properties of polyphenol compounds isolated from the leaves of $M$. communis L. propagated in vitro.

According to Tattini et al. (2006) and Agati et al. (2011), the content of polyphenols, particularly flavonoids, may be related to the reaction of plants to particular environmental conditions, such as light conditions. Light emitting diodes (LED) lighting has great potential for plant in vitro propagation and production. There are many reports confirming LED advantages compared to traditional horticultural lighting (such as incandescent, fluorescent, high-pressure sodium or metal-halide lamps) (Nhut et al. 2003; Gupta and Jatothu 2013). Durability, small size, low heat emission and energy efficiency makes them ideal for the in vitro growing environment (Alvarenga et al. 2015). However, the most important factor is that LED wavelength is much narrower than in traditional light sources. Therefore, a specific and more precise spectral quality can be selected and adjusted to the requirements of a particular plant (Massa et al. 2008; Silva et al. 2017). The appropriate blue and red light ratio seems to be most essential for plant growth. Many researchers have reported the effects of red or blue light on plant morphogenesis and metabolic processes (Kim et al. 2004; Li et al. 2013; Alvarenga et al. 2015), including ornamental species, such as Lilium (Lian et al. 2002), Chrysanthemum (Kim et al. 2004; Kurilčik et al. 2008), Tripterospermum japonicum (Moon et al. 2006) and Dendrobium officinale (Lin et al. 2011).

The objectives of this study were to determine how the growth of $M$. communis L. plantlets and their secondary metabolite contents were affected by the light source (different LED spectra vs. fluorescent lamps) in combination with varying 6-benzyladenine (BA) cytokinin concentrations in media.

\section{Materials and methods}

\section{Plant material}

Potted plants of M. communis L. growing in Department of Ornamental Plants University of Agriculture in Krakow greenhouse collection provided shoot tips for in vitro culture. Axillary shoots were multiplied on Murashige and Skoog (1962) medium (MS) containing $5 \mu \mathrm{M}$ 6-benzyladenine (BA), $0.5 \mu \mathrm{M}$ 1-naphthaleneacetic acid (NAA), $30 \mathrm{~g} \mathrm{dm}^{-3}$ sucrose and $0.5 \%$ BioAgar. The $\mathrm{pH}$ was adjusted to 5.7. Multiplied plantlets were used for the experiment.

\section{Culture and light treatments}

Axillary shoots of $M$. communis (10 mm in height) were cultured on a basal medium (BM) containing MS minerals and vitamins, $30 \mathrm{~g} \mathrm{dm}^{-3}$ sucrose and $0.5 \mu \mathrm{M}$ NAA. BM was gelled with $0.5 \%$ BioAgar and supplemented with three BA concentrations: $1,2.5$ and $5 \mu \mathrm{M}$. The medium was distributed into Erlenmeyer flasks (size $250 \mathrm{ml}$ ) as $30 \mathrm{ml}$ in each one, sealed with aluminium foil and autoclaved at $121{ }^{\circ} \mathrm{C}$ for $21 \mathrm{~min}$. Plant material was treated with four different light quality combinations: B-100\% blue LEDs (peak at $430 \mathrm{~nm}$ ); RB - a mix of $70 \%$ red and $30 \%$ blue LEDs; $\mathrm{R}-100 \%$ red LEDs (peak at $670 \mathrm{~nm}$ ); and $\mathrm{C}$-fluorescent lamp (Philips TL-D 36W/54) as control (Fig. 1). The experiment was conducted in two replications with six repetitions, with six explants each (in total 864 explants). Cultures were maintained for 6 weeks in a growth chamber at $23 / 21 \pm 1{ }^{\circ} \mathrm{C}$ (day/night), $80 \%$ relative humidity and different light sources; $16 / 8 \mathrm{~h}$ photoperiod (day/night) was used and PPFD was kept constant at $35 \mu \mathrm{mol} \mathrm{m}{ }^{-2} \mathrm{~s}^{-1}$ in all treatments.

\section{Data collection}

Biometrical observations comprised shoot multiplication rate, shoot height and number of leaves per shoot, which were recorded after a 6-week cycle. Photographic documentation of plantlet growth and development was made (Sony CyberShot DSCH200, China).

Fresh weight $(\mathrm{FW})$ was determined immediately after removing the plants from vessels. The whole developed plant was weighed by Agrogen (Freibourg, Switzerland).

Multiplied plantlets were oven-dried in an air steriliser at $40{ }^{\circ} \mathrm{C}$ for 4 days to determine dry weight (DW) (Sanyo Electric Co MOV-112S).

For photosynthetic pigment content measurements, 200-mg samples of cut leaves were subjected to the extraction procedure according to methods of Lichtenthaler and Buschmann (2001) and dissolved in $80 \%$ acetone. The absorbance was measured using a UV/VIS Helios Alpha spectrophotometer (Unicam Ltd., Cambridge, UK). The content of chlorophyll $a, b$ and carotenoids was measured at the following wavelength maxima $\left(\mathrm{A}_{\max }\right)$ : chlorophyll $a-663.2 \mathrm{~nm}$, chlorophyll $b-646.8 \mathrm{~nm}$, total carotenoids $-470 \mathrm{~nm}$. The concentration of photosynthetic pigments was calculated using the following formulas: chlorophyll $a\left(c_{a}\right)(\mu \mathrm{g} / \mathrm{ml})=12.25 A_{663.2}-2.79 A_{646.8} ;$ chlorophyll $b\left(c_{b}\right)(\mu \mathrm{g} / \mathrm{ml})=21.50 A_{646.8}-5.10 A_{663.2} ;$ carotenoids $(\mu \mathrm{g} /$ 
Fig. 1 Different LED wavelengths and control fluorescent lamp tested in the experiment: $C$ control, fluorescence Philips TK-D 36W/54 lamps; B 100\% blue LED; $R B \quad 70 \%$ red LED and $30 \%$ blue LED; $R$ 100\% red LED

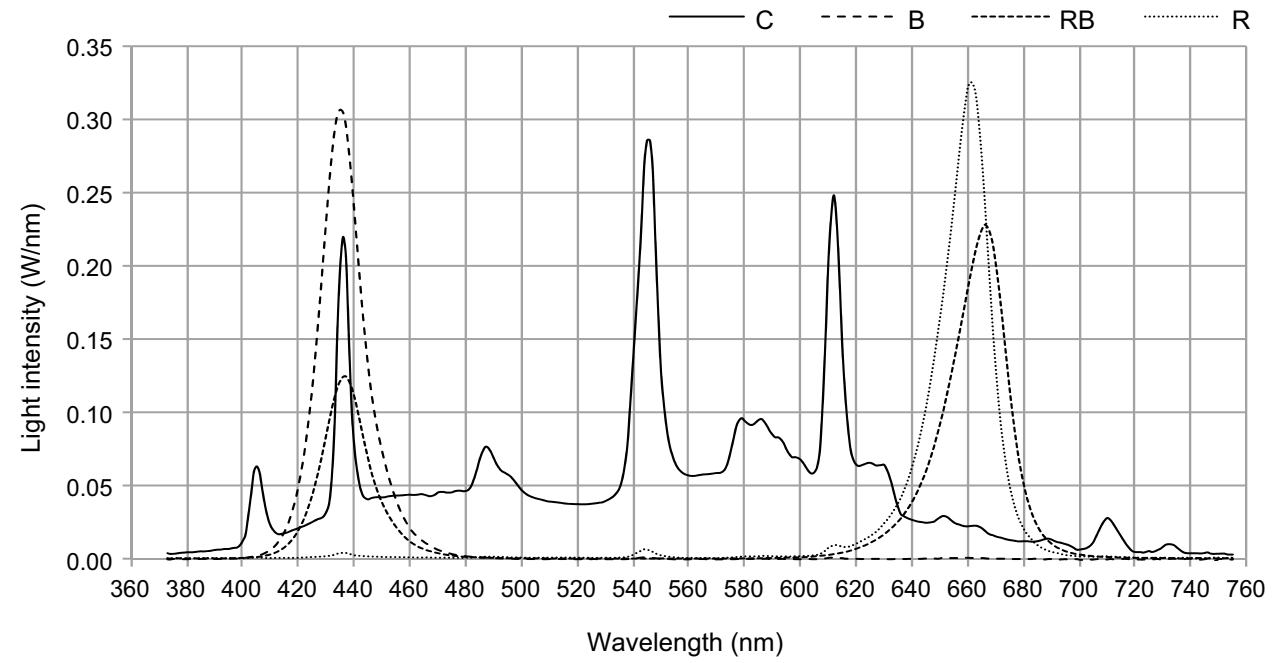

$\mathrm{ml})=\left(1000 A_{470}-1.82 c_{a}-85.02 c_{b}\right) / 198$ (where $A$ is the absorption level) (Lichtenthaler and Buschmann 2001).

RP-HPLC analyses were conducted to measure phenolic acid and flavonoid contents in 0.5-g samples of dry biomass. Phenolic acids and flavonoids were quantified in methanol extracts (sonication, $30{ }^{\circ} \mathrm{C}, 1 \mathrm{~h}$ ) after hydrolysis in $2 \mathrm{M}$ aqueous $\mathrm{HCl}, 100{ }^{\circ} \mathrm{C}, 1 \mathrm{~h}$ (Harborne 1998). RPHPLC analyses were conducted according to the method described elsewhere (Ellnain-Wojtaszek and Zgórka 1999) with our modifications for a Merck-Hitachi liquid chromatograph (LaChrom Elite) equipped with a DAD detector L-2455 and Purospher ${ }^{\circledR}$ RP-18e $(250 \times 4 \mathrm{~mm} / 5 \mu \mathrm{m})$ column. Analyses were carried out at $25^{\circ} \mathrm{C}$, with a mobile phase consisting of $\mathrm{A}$-methanol, $\mathrm{B}$-methanol: $0.5 \%$ acetic acid 1:4 (v/v). The gradient was as follows: $100 \%$ B for 0-20 min; $100-80 \%$ B for 20-35 min; $80-60 \%$ B for 35-55 min; $60-0 \%$ B for 55-70 min; 0\% B for 70-75 min; 0-100\% B for 75-80 min; 100\% B for 80-90 min at a flow rate of $1 \mathrm{ml} \mathrm{min}^{-1}, \lambda=254 \mathrm{~nm}$ (phenolic acids, catechins), $\lambda=370 \mathrm{~nm}$ (flavonoids). The identification was carried out by comparing peak retention times with authentic reference compounds and co-chromatography with standards. Quantification was performed by peak area measurements with reference to the standard curve derived from five concentrations $\left(0.03125-0.5 \mathrm{mg} \mathrm{ml}^{-1}\right)$. Standards for caffeic, chlorogenic, cinnamic, gallic, gentizic, o-coumaric, protocatechuic, salicylic, sinapic and syringic acids, and apigetrin (apigenin 7-glucoside), hyperoside (quercetin 3-galactoside), isorhamnetin, kaempferol, luteolin, populnin (kaemferol 7-O-glucoside), quercetin, quercitrin, rhamnetin, rutin and vitexin were purchased from Sigma Aldrich, while $p$-coumaric, vanillic, ferulic and $p$-hydroxybenzoic acids were purchased from Fluka, and catechin, epigallocatechin, epicatechin gallate, epicatechin, epigallocatechin gallate, cinaroside (luteolin 7-O-glucoside) were from ChromaDex.

\section{Statistical analysis}

The results of the experiment were subjected to ANOVA using Statistica 12 software (StatSoft). The effects of light quality, benzyladenine content and interactions between them were evaluated at three levels of significance: $p \leq 0.05$ $(*), p \leq 0.01(* *)$ and $p \leq 0.001(* * *)$. A Duncan post-hoc multiple range test was used for mean separation and to provide homogeneous groups for the means (at $p \leq 0.05$ ). Standardised data were subjected to multivariate analysis, i.e., k-means clustering and ellipse fitting and principal component analysis (PCA), using Statistica software, for the assessment of chemical composition diversity of $M$. communis plants treated with various light sources and BA concentrations. The eigenvalues were: 3.804, 1.447, 1.266, and below 1 for the remaining PCs. The first two principal components together explained $65.7 \%$ of the total variance, and they were included in the discussion. Correlation-based PCA of chemical constituents (including secondary metabolites and DW) contained in $M$. communis plants was performed. Multiple regression analysis was also conducted, using a stepwise backward elimination method to determine which variables (secondary metabolites and chlorophylls) were most closely related to myricetin concentration. A simplified regression equation was developed; determination coefficient $\left(R^{2}\right)$, adjusted determination coefficient $\left(R_{a d j}^{2}\right)$ as well as standard estimation error (SEE) were determined, with a significance level at $p \leq 0.05$.

\section{Results}

Light spectrum and BA content in media affected the biometrical properties of $M$. communis L. during micropropagation (Table 1; Fig. 2). Shoot multiplication rate ranged from 2.94 to 12.56 and was highest under red (R) LED at a BA 
Table 1 Effect of light quality and cytokinin BA concentration on growth and development of $M$. communis L. from in vitro culture

\begin{tabular}{|c|c|c|c|c|c|c|}
\hline \multirow[t]{2}{*}{ Light quality } & \multirow[t]{2}{*}{$\mathrm{BA}(\mu \mathrm{M})$} & \multirow[t]{2}{*}{ Shoot multiplication rate } & \multirow[t]{2}{*}{ Shoot height $(\mathrm{cm})$} & \multirow[t]{2}{*}{ Mean number of leaves } & \multicolumn{2}{|c|}{ Plantlet weight (mg) } \\
\hline & & & & & Fresh & Dry \\
\hline \multirow[t]{3}{*}{$\mathrm{C}^{\mathrm{b}}$} & 1 & $8.14 \pm 2.44 \mathrm{~cd}^{\mathrm{a}}$ & $8.39 \pm 1.83 \mathrm{~cd}$ & $3.89 \pm 0.53 \mathrm{a}$ & $200.0 \pm 36.83 \mathrm{ab}$ & $33.07 \pm 4.33 \mathrm{ab}$ \\
\hline & 2.5 & $6.75 \pm 1.04 \mathrm{a}-\mathrm{d}$ & $8.55 \pm 2.90 \mathrm{~cd}$ & $4.92 \pm 0.74 \mathrm{a}$ & $154.2 \pm 13.767 \mathrm{a}$ & $27.45 \pm 1.67 \mathrm{ab}$ \\
\hline & 5 & $8.52 \pm 2.10 \mathrm{~cd}$ & $6.00 \pm 2.29 \mathrm{a}-\mathrm{c}$ & $5.17 \pm 1.59 \mathrm{a}$ & $185.0 \pm 57.66 \mathrm{ab}$ & $42.33 \pm 9.43 \mathrm{a}-\mathrm{c}$ \\
\hline \multirow[t]{3}{*}{$\mathrm{B}$} & 1 & $9.48 \pm 0.98 \mathrm{de}$ & $8.94 \pm 1.83 \mathrm{~cd}$ & $4.94 \pm 0.91 \mathrm{a}$ & $324.2 \pm 31.26 \mathrm{c}$ & $43.30 \pm 4.86 \mathrm{a}-\mathrm{c}$ \\
\hline & 2.5 & $7.33 \pm 1.50 \mathrm{~b}-\mathrm{d}$ & $6.39 \pm 3.39 \mathrm{a}-\mathrm{d}$ & $4.43 \pm 0.55 \mathrm{a}$ & $250.8 \pm 51.32 \mathrm{bc}$ & $32.43 \pm 5.86 \mathrm{ab}$ \\
\hline & 5 & $3.81 \pm 1.10 \mathrm{ab}$ & $3.86 \pm 1.50 \mathrm{a}$ & $13.88 \pm 4.17 \mathrm{~b}$ & $251.7 \pm 41.56 \mathrm{bc}$ & $52.65 \pm 13.86 \mathrm{bc}$ \\
\hline \multirow[t]{3}{*}{$\mathrm{RB}$} & 1 & $7.16 \pm 2.99 \mathrm{~b}-\mathrm{d}$ & $7.77 \pm 1.67 \mathrm{~b}-\mathrm{d}$ & $3.99 \pm 1.43 \mathrm{a}$ & $247.5 \pm 33.63 \mathrm{bc}$ & $27.24 \pm 3.56 \mathrm{ab}$ \\
\hline & 2.5 & $5.17 \pm 1.48 \mathrm{a}-\mathrm{c}$ & $7.01 \pm 2.19 \mathrm{a}-\mathrm{d}$ & $5.44 \pm 1.48 \mathrm{a}$ & $207.2 \pm 26.58 \mathrm{ab}$ & $30.59 \pm 3.71 \mathrm{ab}$ \\
\hline & 5 & $2.94 \pm 2.79 \mathrm{a}$ & $4.72 \pm 1.67 \mathrm{ab}$ & $11.19 \pm 5.80 \mathrm{~b}$ & $260.8 \pm 90.50 \mathrm{bc}$ & $59.41 \pm 18.69 \mathrm{~cd}$ \\
\hline \multirow[t]{3}{*}{$\mathrm{R}$} & 1 & $10.33 \pm 2.64 \mathrm{de}$ & $12.56 \pm 3.87 \mathrm{e}$ & $4.54 \pm 0.48 \mathrm{a}$ & $213.3 \pm 8.04 \mathrm{ab}$ & $21.71 \pm 2.17 \mathrm{a}$ \\
\hline & 2.5 & $9.20 \pm 3.40 \mathrm{c}-\mathrm{e}$ & $9.49 \pm 3.38 \mathrm{~d}$ & $3.58 \pm 1.01 \mathrm{a}$ & $184.2 \pm 32.63 \mathrm{ab}$ & $23.81 \pm 2.21 \mathrm{a}$ \\
\hline & 5 & $12.56 \pm 3.10 \mathrm{e}$ & $7.97 \pm 2.79 b-d$ & $4.23 \pm 1.49 \mathrm{a}$ & $267.5 \pm 61.44 \mathrm{bc}$ & $74.45 \pm 38.00 \mathrm{~d}$ \\
\hline \multicolumn{7}{|l|}{ Means for light quality } \\
\hline $\mathrm{C}$ & & $7.80 \pm 3,69 b$ & $7.65 \pm 2.54 \mathrm{a}$ & $4.66 \pm 1.14 \mathrm{a}$ & $179.7 \pm 40.34 \mathrm{a}$ & $33.87 \pm 8.06 \mathrm{a}$ \\
\hline B & & $6.87 \pm 3,42 \mathrm{ab}$ & $6.40 \pm 3.09 \mathrm{a}$ & $7.75 \pm 3.32 b$ & $275.6 \pm 51.61 \mathrm{c}$ & $42.91 \pm 11.99 \mathrm{a}$ \\
\hline $\mathrm{RB}$ & & $5,09 \pm 2,94 \mathrm{a}$ & $6.50 \pm 2.20 \mathrm{a}$ & $6.87 \pm 2.62 b$ & $238.5 \pm 55.60 \mathrm{bc}$ & $38.89 \pm 17.94 \mathrm{a}$ \\
\hline $\mathrm{R}$ & & $10.69 \pm 3,22 \mathrm{c}$ & $10.01 \pm 3.73 b$ & $4.11 \pm 1.09 \mathrm{a}$ & $221.7 \pm 50.67 \mathrm{ab}$ & $40.84 \pm 33.37 \mathrm{a}$ \\
\hline \multicolumn{7}{|l|}{ Means for BA } \\
\hline 1 & & $8.78 \pm 2,55 \mathrm{a}$ & $9.41 \pm 2.99 \mathrm{c}$ & $4.34 \pm 0.96 \mathrm{a}$ & $246.3 \pm 56.36 \mathrm{~b}$ & $31.25 \pm 8.98 \mathrm{a}$ \\
\hline 2.5 & & $7.11 \pm 2,42 \mathrm{a}$ & $7.86 \pm 3.07 \mathrm{~b}$ & $4.59 \pm 1.17 \mathrm{a}$ & $199.1 \pm 46.85 \mathrm{a}$ & $28.44 \pm 4.73 \mathrm{a}$ \\
\hline 5 & & $6.96 \pm 3,58 \mathrm{a}$ & $5.64 \pm 2.53 \mathrm{a}$ & $8.61 \pm 3.32 b$ & $241.3 \pm 65.41 \mathrm{~b}$ & $57.70 \pm 23.69 \mathrm{~b}$ \\
\hline \multicolumn{7}{|l|}{ Source of variation } \\
\hline Light quality × BA & & $*$ & $* * *$ & $* *$ & $* * *$ & $* * *$ \\
\hline Light quality & & $* * *$ & $* * *$ & $* *$ & $* *$ & $\mathrm{n} . \mathrm{s}$ \\
\hline BA & & n.s & $* * *$ & $* * *$ & $*$ & $* * *$ \\
\hline
\end{tabular}

Significant effect: $* p \leq 0.05 ; * * \leq 0.01 ; * * p \leq 0.001 ; n . s$. not significant

${ }^{a}$ Means \pm standard deviations within a column followed by the same letter are not significantly different according to Duncan's multiple range test at $p \leq 0.05$

${ }^{\mathrm{b}} C$ control, fluorescence Philips TK-D 36W/54 lamps; $B$ 100\% blue LED; $R B 70 \%$ red LED and 30\% blue LED; $R$ 100\% red LED

concentration of $5 \mu \mathrm{M}(12.56)$ and 1 and $2.5 \mu \mathrm{M}$ (10.33 and 9.20, respectively) (Fig. 2j-1). A high rate was also obtained at $1 \mu \mathrm{M}$ BA under blue (B) LED (9.48) (Fig. 2d). It was found that under the $\mathrm{B}$ LED light spectrum [monochromatic or with red (i.e. RB)], the increase in BA had an inhibitory effect on the micropropagation rate. Considering the effect of light irrespective of the cytokinin content, it can be stated that the monochromatic red LED light stimulated the multiplication rate. If blue light was present (B or $\mathrm{RB}$ ), then the multiplication rate was lower.

Shoots grew the highest at lower cytokinin concentrations in the medium and under red LED (Table 1; Fig. 2j-1); R stimulated shoot height (regardless of cytokinin content). The more cytokinin there was in the medium, the shorter the shoots were, especially under B light.

Shoots had the most leaves when they were propagated under light emitting diodes containing blue waves (B or $\mathrm{RB})$ in media with the highest BA concentration. This was confirmed by the main effect analysis-M. communis under blue LED diodes (B and RB) had significantly more leaves in comparison to other treatments. Plants growing on the medium with the highest cytokinin concentration had significantly more leaves.

Plantlet FW ranged from 154.2 to $324.2 \mathrm{mg}$. Light as the main factor influenced the level of FW-the highest FW was obtained under B or RB LEDs compared to control. Plant FW was the highest at extreme cytokinin concentrations in the medium $(1$ or $5 \mu \mathrm{M})$. The highest BA content $(5 \mu \mathrm{M})$ in the media increased DW the most, on average by $85-103 \%$, in comparison to the plants micropropagated with 2.5 and $1 \mu \mathrm{M}$ BA.

Medium composition and the type of light affected photosynthetic pigment contents (Fig. 3). Chlorophyll $a$ level ranged from 0.36 to $1.02 \mathrm{mg} \mathrm{g}^{-1} \mathrm{FW}$, chlorophyll $b$ was $0.13-0.32 \mathrm{mg} \mathrm{g}^{-1} \mathrm{FW}$, and carotenoids were found at $0.13-0.33 \mathrm{mg} \mathrm{g}^{-1} \mathrm{FW}$. Generally, there was less 

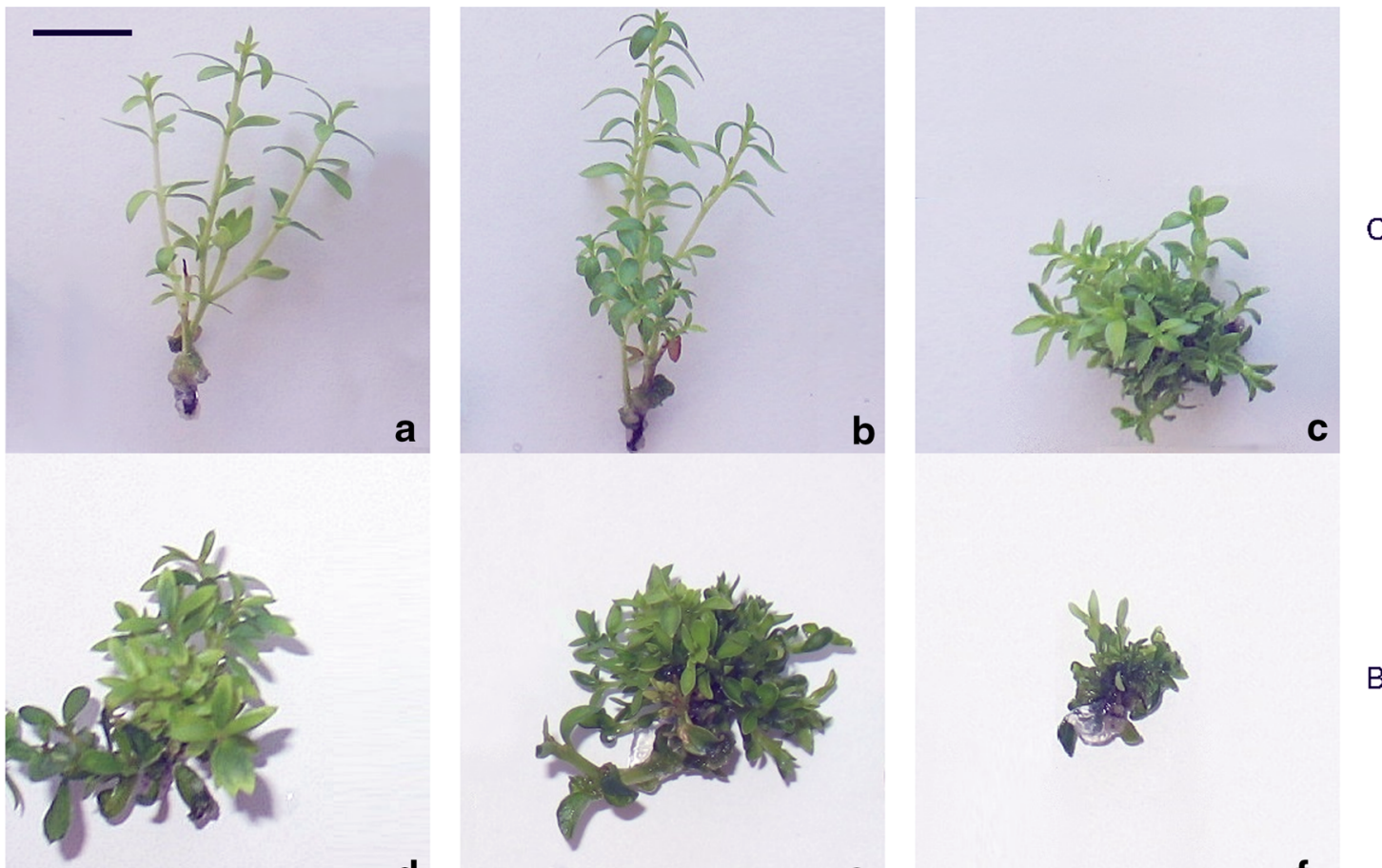

d

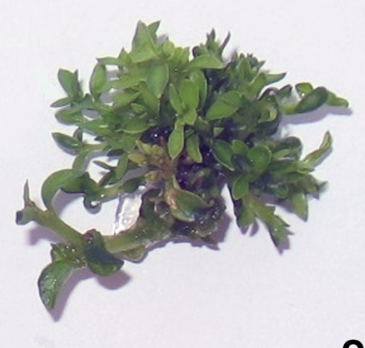

e

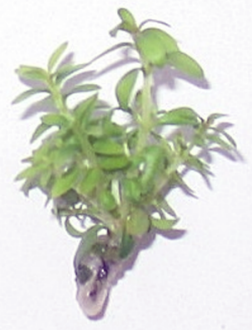

h

RB

g

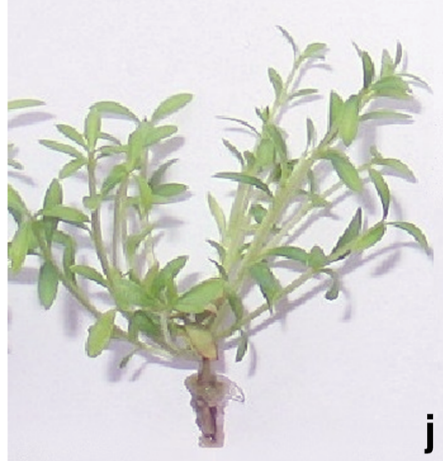

$1 \mu \mathrm{M}$

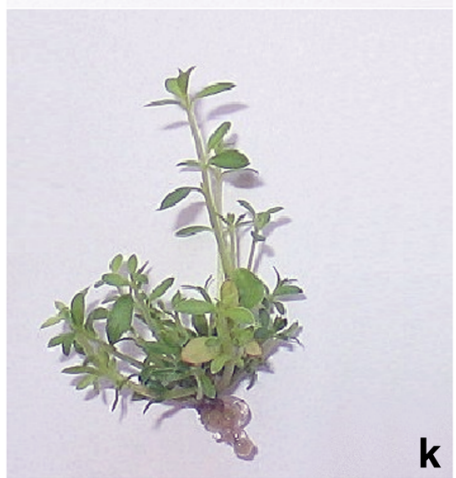

$2.5 \mu \mathrm{M}$

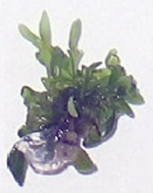

B

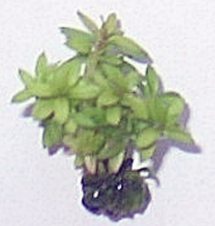

C

f

$\mathrm{R}$

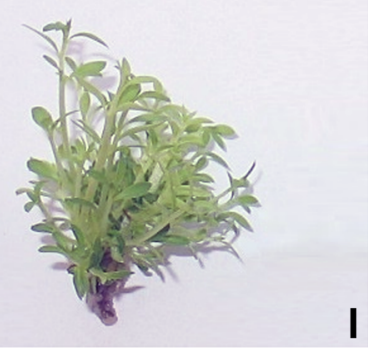

$5 \mu \mathrm{M}$

BA

Fig. 2 Growth and development of $M$. communis after 6 weeks of culture under different light qualities. a-c control, fluorescence Philips TK-D 36W/54 lamps (C); d-f 100\% blue LED (B); g-i 70\% red LED and 30\% blue LED (RB); $\mathbf{j}-\mathbf{l} 100 \%$ red LED (R), and various BA content in media. $\mathrm{Bar}=2 \mathrm{~cm}$ 
a
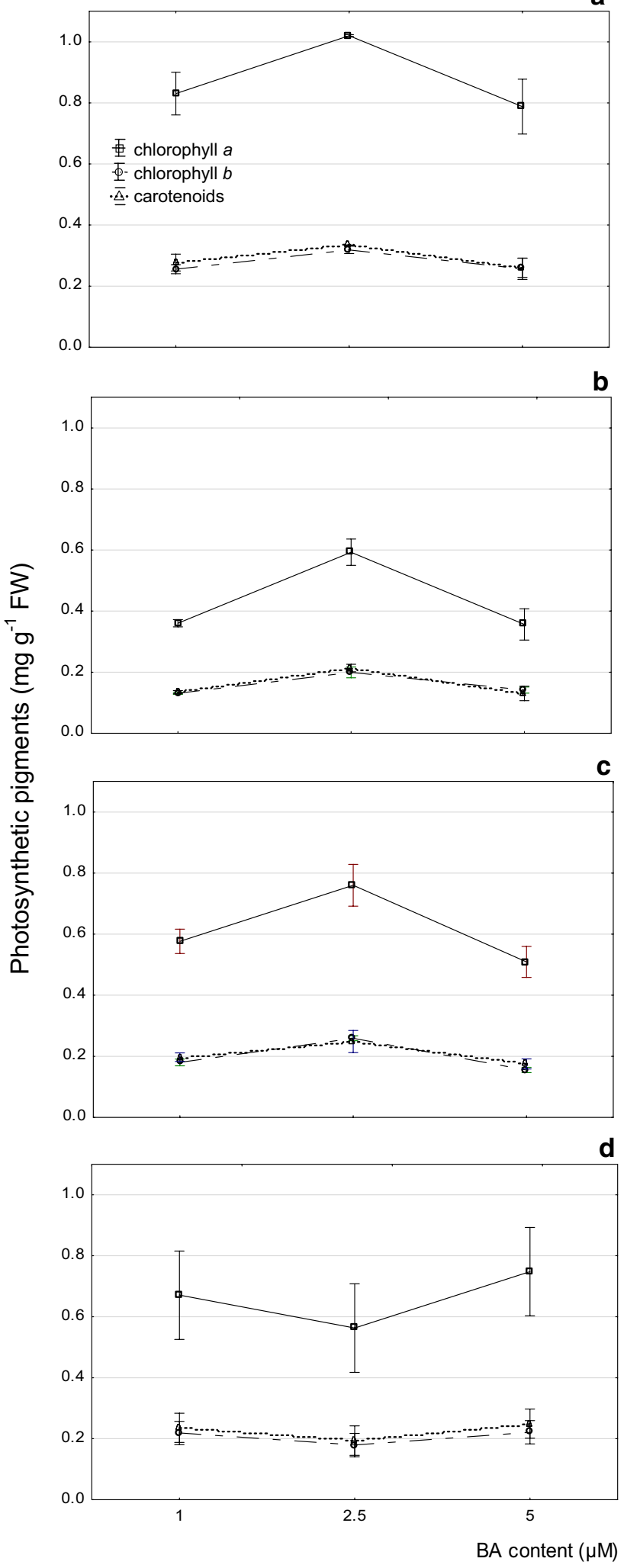

Fig. 3 Effect of different light qualities and BA content on photosynthetic pigment concentrations in $M$. communis in vitro. a Control, fluorescence Philips TK-D 36W/54 lamps (C); b 100\% blue LED (B); c $70 \%$ red LED and $30 \%$ blue LED (RB); d $100 \%$ red LED (R) and various $\mathrm{BA}$ content in media photosynthetic pigment under LED lights than under control (C) fluorescent light. The lowest amount of photosynthetic pigment was recorded under B light. The highest photosynthetic pigment contents were always on $2.5 \mu \mathrm{M}$ BA medium, except for the R LED, where the highest amount of pigment was found on $5 \mu \mathrm{M}$ BA media.

Among the secondary metabolites present in methanol extracts of M. communis leaves, we identified the presence of phenolic acids, such as gallic, protocatechuic and $p$-hydroxybenzoic acids by using the above-listed standards. Of other polyphenolic compounds, we found flavonoids, such as myricetin, catechin, and its derivatives, epigallocatechin and epigallocatechin gallate. Of the polyphenols found, $M$. communis leaves contained the highest amounts of myricetin (347.02-1118.69 mg $100 \mathrm{~g}^{-1} \mathrm{DW}$ ). Table 2 shows that the concentration of BA in the medium, the light spectrum and the interaction of these experimental factors had a significant effect on the content of secondary metabolites. The highest content of flavonoids was observed in the medium with a 5- $\mu \mathrm{M}$ BA concentration.

The highest flavonoid contents were always observed under R light, when only the effect of light on the content of secondary metabolites in $M$. communis leaf extracts was considered. The lowest amount of catechins and epigallocatechins were found in M. communis under fluorescent lamps, whereas the level of myricetin and epigallocatechin gallate decreased due to the use of blue diodes. Analysing in detail the interactions of the medium with light type, the lowest content of the flavonoids, epigallocatechin gallate and myricetin, was observed in a $2.5-\mu \mathrm{M}$ medium and blue light, while less catechin and epigallocatechin was recorded under fluorescent light. The highest amounts of catechin and its derivatives as well as myricetin were found in M. communis produced on a 5- $\mu \mathrm{M}$ medium and $\mathrm{R}$ light.

Of the phenolic acids, the highest concentrations were observed for gallic acid (95.58 $\mathrm{mg} 100 \mathrm{~g}^{-1} \mathrm{DW}$ on average), while the remaining protocatechic and $p$-hydroxybenzoic acids amounted to 2.59 and $5.13 \mathrm{mg} 100 \mathrm{~g}^{-1} \mathrm{DW}$, respectively. The highest content of gallic acid was observed under $\mathrm{R}$ LED and $\mathrm{C}$ light on the medium containing $5 \mu \mathrm{M}$ BA. The highest amount of protocatechuic acid was found in $M$. communis under RB diodes and $1 \mu \mathrm{M} \mathrm{BA}$, while the highest level of $p$-hydroxybenzoic acid was found in plantlets in $\mathrm{C}$ with $\mathrm{BA}$ content in media equal to $1 \mu \mathrm{M}$. Considering the main effect of light quality, it is important to highlight the lowest phenolic acid contents were found under B light. LED light reduced the gallic acid content, while the highest protocatechuic acid content was found in M. communis under RB light, while for $p$-hydroxybenzoic acid, the highest concentration was found under $\mathrm{R}$ light. The lowest amounts of phenolic acids were observed on media containing $2.5 \mu \mathrm{M}$ BA. 
Table 2 Effect of light quality and cytokinin BA concentration on secondary metabolites ( $\mathrm{mg} 100 \mathrm{~g}^{-1}$ dry weight) in the leaves of M. communis L. from in vitro culture

\begin{tabular}{|c|c|c|c|c|c|c|c|c|}
\hline \multirow{3}{*}{$\begin{array}{l}\text { Light qual- } \\
\text { ity }\end{array}$} & \multirow[t]{3}{*}{$\mathrm{BA}(\mu \mathrm{M})$} & \multicolumn{3}{|l|}{ Before hydrolysis } & \multicolumn{4}{|l|}{ After hydrolysis } \\
\hline & & \multicolumn{4}{|l|}{ Flavonoids } & \multicolumn{3}{|l|}{ Phenolic acids } \\
\hline & & Catechin & $\begin{array}{l}\text { Epigallocat- } \\
\text { echin }\end{array}$ & $\begin{array}{l}\text { Epigallocat- } \\
\text { echin gallate }\end{array}$ & Myricetin & Gallic acid & $\begin{array}{l}\text { Protocat- } \\
\text { echuic acid }\end{array}$ & $\begin{array}{l}p \text {-hydroxy- } \\
\text { benzoic acid }\end{array}$ \\
\hline \multirow[t]{3}{*}{$C^{b}$} & 1 & $51.62 \pm 2.46 \mathrm{a}^{\mathrm{a}}$ & $29.95 \pm 0.86 \mathrm{a}$ & $13.67 \pm 0.37 \mathrm{c}$ & $680.93 \pm 14.82 \mathrm{~g}$ & $152.37 \pm 0.61 \mathrm{~h}$ & $0.01 \pm 0.00 \mathrm{a}$ & $16.92 \pm 0.18 \mathrm{j}$ \\
\hline & 2.5 & $55.42 \pm 3.42 \mathrm{ab}$ & $40.20 \pm 4.27 \mathrm{a}$ & $14.22 \pm 0.54 \mathrm{~cd}$ & $713.02 \pm 6.38 \mathrm{~h}$ & $139.57 \pm 1.22 \mathrm{~g}$ & $3.50 \pm 0.27 \mathrm{~d}$ & $0.38 \pm 0.04 \mathrm{a}$ \\
\hline & 5 & $86.46 \pm 3.45$ e & $109.82 \pm 4.79 \mathrm{e}$ & $11.28 \pm 0.06 \mathrm{~b}$ & $618.13 \pm 12.61 \mathrm{e}$ & $172.36 \pm 0.61 \mathrm{j}$ & $3.44 \pm 0.24 \mathrm{~d}$ & $1.43 \pm 0.10 \mathrm{~b}$ \\
\hline \multirow[t]{3}{*}{ B } & 1 & $77.22 \pm 1.93 \mathrm{~d}$ & $52.12 \pm 0.98 \mathrm{~b}$ & $12.06 \pm 0.11 \mathrm{~b}$ & $395.44 \pm 8.60 \mathrm{~b}$ & $72.11 \pm 0.10 \mathrm{f}$ & $2.76 \pm 0.01 \mathrm{c}$ & $1.03 \pm 0.01 \mathrm{~b}$ \\
\hline & 2.5 & $58.02 \pm 2.61 \mathrm{~b}$ & $72.39 \pm 2.18 \mathrm{c}$ & $3.41 \pm 0.02 \mathrm{a}$ & $347.02 \pm 0.11 \mathrm{a}$ & $15.51 \pm 0.31 \mathrm{a}$ & $0.01 \pm 0.00 \mathrm{a}$ & $1.05 \pm 0.08 \mathrm{~b}$ \\
\hline & 5 & $96.87 \pm 5.51 \mathrm{f}$ & $133.24 \pm 15.20 \mathrm{f}$ & $14.78 \pm 0.17 \mathrm{de}$ & $658.97 \pm 6.75 \mathrm{f}$ & $19.41 \pm 0.36 b$ & $0.10 \pm 0.00 \mathrm{a}$ & $4.05 \pm 0.02 \mathrm{~d}$ \\
\hline \multirow[t]{3}{*}{$\mathrm{RB}$} & 1 & $63.54 \pm 3.84 \mathrm{c}$ & $52.66 \pm 1.23 \mathrm{~b}$ & $26.70 \pm 0.23 \mathrm{~g}$ & $458.46 \pm 5.66 \mathrm{c}$ & $166.82 \pm 1.51 \mathrm{i}$ & $7.47 \pm 0.59 \mathrm{f}$ & $3.22 \pm 0.04 \mathrm{c}$ \\
\hline & 2.5 & $73.63 \pm 4.57 \mathrm{~d}$ & $60.71 \pm 1.29 b$ & $18.49 \pm 0.59 \mathrm{f}$ & $551.46 \pm 4.78 \mathrm{~d}$ & $43.65 \pm 0.19 \mathrm{e}$ & $5.30 \pm 0.00 \mathrm{e}$ & $5.20 \pm 0.27 \mathrm{f}$ \\
\hline & 5 & $85.11 \pm 0.13 \mathrm{e}$ & $112.26 \pm 9.07 \mathrm{e}$ & $15.64 \pm 0.03 \mathrm{e}$ & $750.57 \pm 7.85 \mathrm{i}$ & $73.17 \pm 0.31 \mathrm{f}$ & $3.08 \pm 0.04 \mathrm{c}$ & $7.81 \pm 0.90 \mathrm{~h}$ \\
\hline \multirow[t]{3}{*}{$\mathrm{R}$} & 1 & $78.43 \pm 0.84 \mathrm{~d}$ & $35.65 \pm 1.97 \mathrm{a}$ & $18.53 \pm 0.73 \mathrm{f}$ & $714.62 \pm 5.72 \mathrm{~h}$ & $42.48 \pm 0.13 \mathrm{~d}$ & $3.52 \pm 0.01 \mathrm{~d}$ & $6.95 \pm 0.04 \mathrm{~g}$ \\
\hline & 2.5 & $100.74 \pm 2.21 \mathrm{f}$ & $99.73 \pm 5.70 \mathrm{~d}$ & $40.75 \pm 1.38 \mathrm{~h}$ & $455.87 \pm 6.05 \mathrm{c}$ & $25.44 \pm 0.66 \mathrm{c}$ & $0.01 \pm 0.00 \mathrm{a}$ & $4.59 \pm 0.04 \mathrm{e}$ \\
\hline & 5 & $132.81 \pm 1.99 \mathrm{~g}$ & $199.26 \pm 3.15 \mathrm{~g}$ & $45.85 \pm 0.94 \mathrm{i}$ & $1118.69 \pm 12.17 \mathrm{j}$ & $224.05 \pm 0.36 \mathrm{k}$ & $1.91 \pm 0.01 \mathrm{~b}$ & $8.89 \pm 0.18 \mathrm{i}$ \\
\hline \multicolumn{9}{|c|}{ Means for light quality } \\
\hline \multicolumn{2}{|l|}{$\mathrm{C}$} & $64.50 \pm 16.78 \mathrm{a}$ & $59.99 \pm 37.77 \mathrm{a}$ & $13.06 \pm 1.39 \mathrm{~b}$ & $670.69 \pm 43.04 \mathrm{c}$ & $154.77 \pm 14.33 \mathrm{~d}$ & $2.31 \pm 1.74 \mathrm{c}$ & $6.24 \pm 8.02 \mathrm{c}$ \\
\hline \multicolumn{2}{|l|}{$\mathrm{B}$} & $77.37 \pm 17.13 \mathrm{c}$ & $85.92 \pm 37.36 \mathrm{c}$ & $10.08 \pm 5.15 \mathrm{a}$ & $467.14 \pm 145.49 \mathrm{a}$ & $35.68 \pm 27.38 \mathrm{a}$ & $0.95 \pm 1.35 \mathrm{a}$ & $2.04 \pm 1.50 \mathrm{a}$ \\
\hline \multicolumn{2}{|l|}{$\mathrm{RB}$} & $74.10 \pm 9.81 \mathrm{~b}$ & $75.21 \pm 28.38 \mathrm{~b}$ & $20.28 \pm 4.98 \mathrm{c}$ & $586.83 \pm 129.35 \mathrm{~b}$ & $94.55 \pm 55.70 \mathrm{~b}$ & $5.28 \pm 1.93 \mathrm{~d}$ & $5.41 \pm 2.04 \mathrm{~b}$ \\
\hline \multicolumn{2}{|l|}{$\mathrm{R}$} & $104.00 \pm 23.72 \mathrm{~d}$ & $111.55 \pm 71.48 \mathrm{~d}$ & $35.04 \pm 12.61 \mathrm{~d}$ & $763.06 \pm 289.39 \mathrm{~d}$ & $97.32 \pm 95.33 \mathrm{c}$ & $1.81 \pm 1.53 b$ & $6.81 \pm 1.86 \mathrm{~d}$ \\
\hline \multicolumn{9}{|c|}{ Means for BA } \\
\hline \multicolumn{2}{|l|}{1} & $67.70 \pm 11.66 \mathrm{a}$ & $42.60 \pm 10.51 \mathrm{a}$ & $17.74 \pm 5.96 \mathrm{a}$ & $562.36 \pm 144.10 \mathrm{~b}$ & $108.45 \pm 54.80 \mathrm{~b}$ & $3.44 \pm 2.80 \mathrm{~b}$ & $7.03 \pm 6.36 \mathrm{c}$ \\
\hline \multicolumn{2}{|l|}{2.5} & $71.95 \pm 19.03 \mathrm{~b}$ & $68.26 \pm 22.70 \mathrm{~b}$ & $19.22 \pm 14.21 \mathrm{~b}$ & $516.84 \pm 140.43 \mathrm{a}$ & $56.04 \pm 51.46 \mathrm{a}$ & $2.20 \pm 2.39 \mathrm{a}$ & $2.81 \pm 2.21 \mathrm{a}$ \\
\hline \multicolumn{2}{|l|}{5} & $100.32 \pm 20.37 \mathrm{c}$ & $138.64 \pm 38.60 \mathrm{c}$ & $21.89 \pm 14.56 \mathrm{c}$ & $786.59 \pm 206.61 \mathrm{c}$ & $122.25 \pm 83.98 \mathrm{c}$ & $2.13 \pm 1.36 \mathrm{a}$ & $5.54 \pm 3.14 \mathrm{~b}$ \\
\hline \multicolumn{9}{|c|}{ Source of variation } \\
\hline \multicolumn{2}{|c|}{ Light quality $\times$ BA } & $* * *$ & $* * *$ & $* * *$ & $* * *$ & $* * *$ & $* * *$ & $* * *$ \\
\hline \multicolumn{2}{|c|}{ Light quality } & $* * *$ & $* * *$ & $* * *$ & $* * *$ & $* * *$ & $* * *$ & $* * *$ \\
\hline \multicolumn{2}{|l|}{ BA } & $* * *$ & $* * *$ & $* * *$ & $* * *$ & $* * *$ & $* * *$ & $* * *$ \\
\hline
\end{tabular}

Significant effect: $* * * p \leq 0.001 ;$ n.s. not significant

a Means \pm standard deviations within a column followed by the same letter are not significantly different according to Duncan's multiple range test at $p \leq 0.05$

${ }^{\mathrm{b}} C$ control, fluorescence Philips TK-D 36W/54 lamps; $B$ 100\% blue LED; $R B$ 70\% red LED and 30\% blue LED; $R$ 100\% red LED

The highest total content of all detected secondary metabolites was observed under R LED (1045.2 mg 100 $\mathrm{g}^{1} \mathrm{DW}$ ), followed by C light (971.6 mg $\left.100 \mathrm{~g}^{-1} \mathrm{DW}\right), \mathrm{RB}$ (862.3 $\left.\mathrm{mg} 100 \mathrm{~g}^{-1} \mathrm{DW}\right)$, and the lowest was found under B LED (674 mg $\left.100 \mathrm{~g}^{-1} \mathrm{DW}\right)$. Plantlets propagated under fluorescent light had more secondary metabolites than under LED with blue wavelength in their spectrum $(B, R B)$. The highest amount of secondary metabolites was synthesised by plantlets in the medium containing $5 \mu \mathrm{M} \mathrm{BA}$. Low contents of secondary metabolites were found in plantlets cultured under B LED and on a 2.5- $\mu \mathrm{M}$ BA medium (summative data not shown).

The resulting score plot provides a conceptual overview of the treatments by showing a total of $65.7 \%$ of the variance (Fig. 4). PC1 separated these treatments, where the BA content of $5 \mu \mathrm{M}$ was distinguished (including one treatment with $100 \% \mathrm{R}$ light and $2.5 \mu \mathrm{M}$ of BA content) from the plants subjected to lower BA concentrations. PC2 separated all treatments with $100 \% \mathrm{~B}$ light, and also R/2.5 and $\mathrm{RB} / 5 \mu \mathrm{M}$, from others. The loads of plants supplemented with $\mathrm{R}$ light and grown on the medium containing $5 \mu \mathrm{M}$ with PC1 was the highest (-5.423), while $\mathrm{RB} / 1 \mu \mathrm{M}$ had the highest negative factor loadings with the second component $(-2.110)$. Taking into account the highest negative loading of PC1, it is possible to conclude that $M$. communis illuminated with 100\% R LED light and grown on the medium with $5 \mu \mathrm{M}$ BA differed from the other treatments by its higher contents of catechin, epigallocatechin, epigallocatechin gallate, gallic acid and myricetin. 
Fig. 4 Score plot of principal components 1 and 2 for 12 experimental treatments: $C$ control, fluorescence Philips TK-D 36W/54 lamps; B 100\% blue LED; $R B \quad 70 \%$ red LED and $30 \%$ blue LED; $R 100 \%$ red LED; BA content- $1,2.5,5 \mu \mathrm{M}$

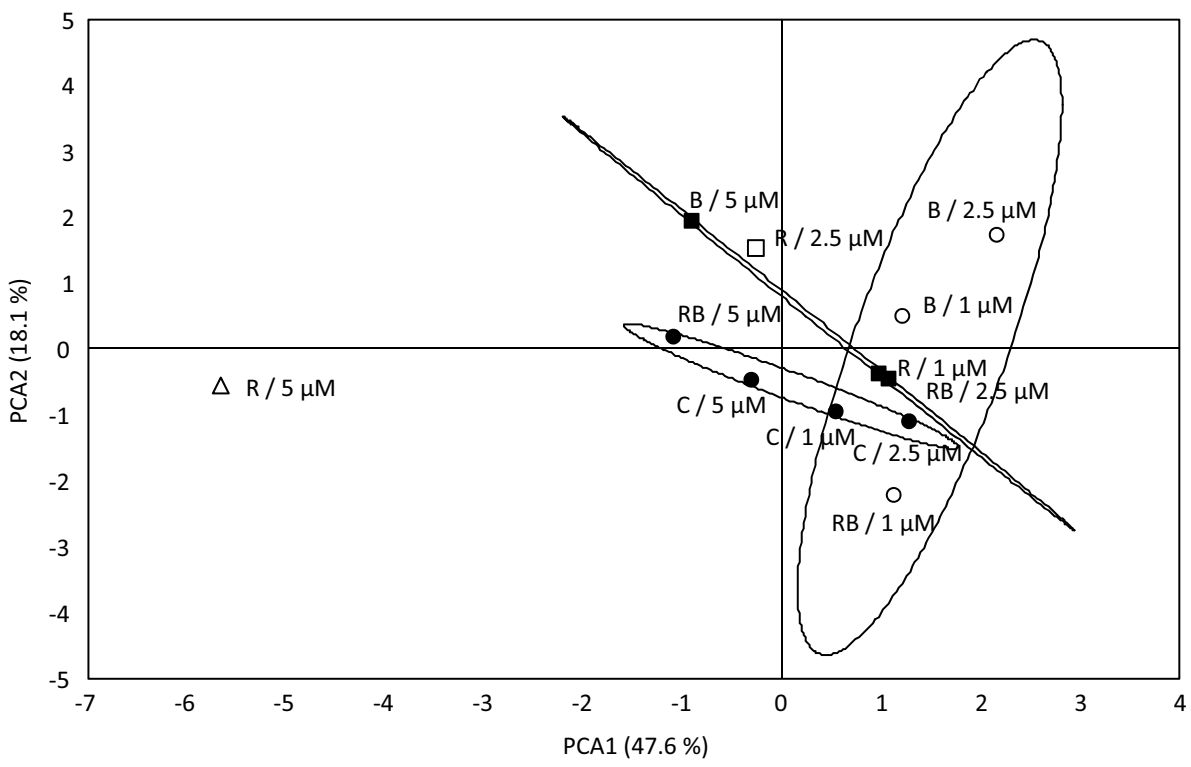

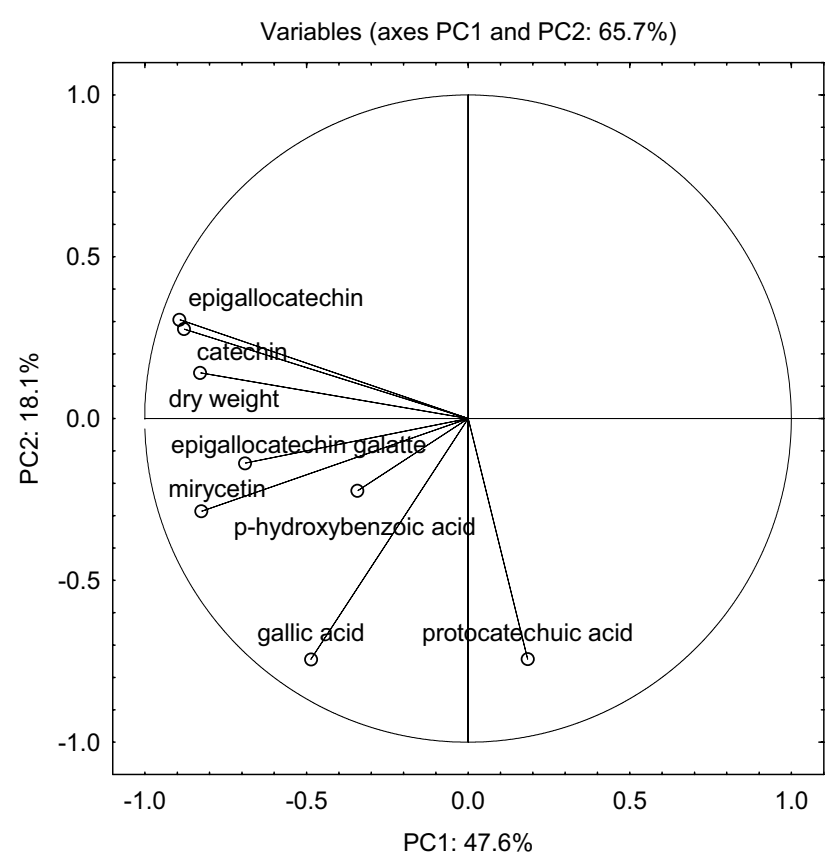

Fig. 5 Correlation circle of the principal components analysis (PCA) on the correlation matrix built using data for dry weight and secondary metabolites

There were several significant relationships between plant constituents (secondary metabolites, DW) (Fig. 5). DW was positively correlated with catechin, epigallocatechin, and myricetin ( $r=0.684 ; 0.835 ; 0.645$, respectively). We also found significant and close relationships between catechin and epigallocatechin $(r=0.896)$ or epigallocatechin gallate $(r=0.718)$. Positive and statistically significant correlations between myricetin and epigallocatechin $(r=0.578)$ was also observed.
Multiple regression analysis confirmed that variations in catechin and chlorophyll $a$ concentrations were primarily responsible for the content of myricetin in $M$. соттиnis plants. Variables of the preliminary regression model included secondary metabolites and chlorophylls (Chl $a$ and $\mathrm{Chl} b$ ). The simplified model obtained through regression multiple analysis contained two independent variables: catechin and chlorophyll $a$ contents. The fitting of the model stood at the level of $62 \%\left(R^{2}=0.623\right)$, hence it follows that the remaining $38 \%$ of the myricetin content variance was dependent on other variables that were not included in the analysis; the adjusted determination coefficient $\left(R_{a d j}^{2}=0.539\right)$ was at an intermediate level $(p \leq 0.012)$. Standard estimation error ( $S E E$ ) was 140.5 , i.e. the equation is accurate to an average of about $140 \mathrm{mg}$ myricetin $100 \mathrm{~g}^{-1}$ DW in estimating myricetin concentration in $\mathrm{M}$. соттиnis plants. Comparison of the observed data and simulated values, calculated from a regression equation, is presented in Fig. 6.

\section{Discussion}

Myrtus communis L. is a very well-known plant for its various properties associated with secondary metabolites. Many works have focused on volatile or phenolic compounds in $M$. communis berries (Alipour et al. 2014; Bajalan and Ghasemi Pirbalouti 2014; Badra et al. 2016), but there are also studies on the antioxidant activity of $M$. communis leaf extracts (Amessis-Ouchemoukh et al. 2014; Bouaziz et al. 2015). To our knowledge, there have been no studies into the effects of light on growth, development and secondary metabolite contents of M. communis cultivated in vitro. In our experiment, plantlets from in vitro conditions were used for biochemical 
Fig. 6 Predicted myricetin content in myrtle plants vs. values observed in the experiment, plotted on the basis of regression model, including catechin and chlorophyll $a$ concentrations

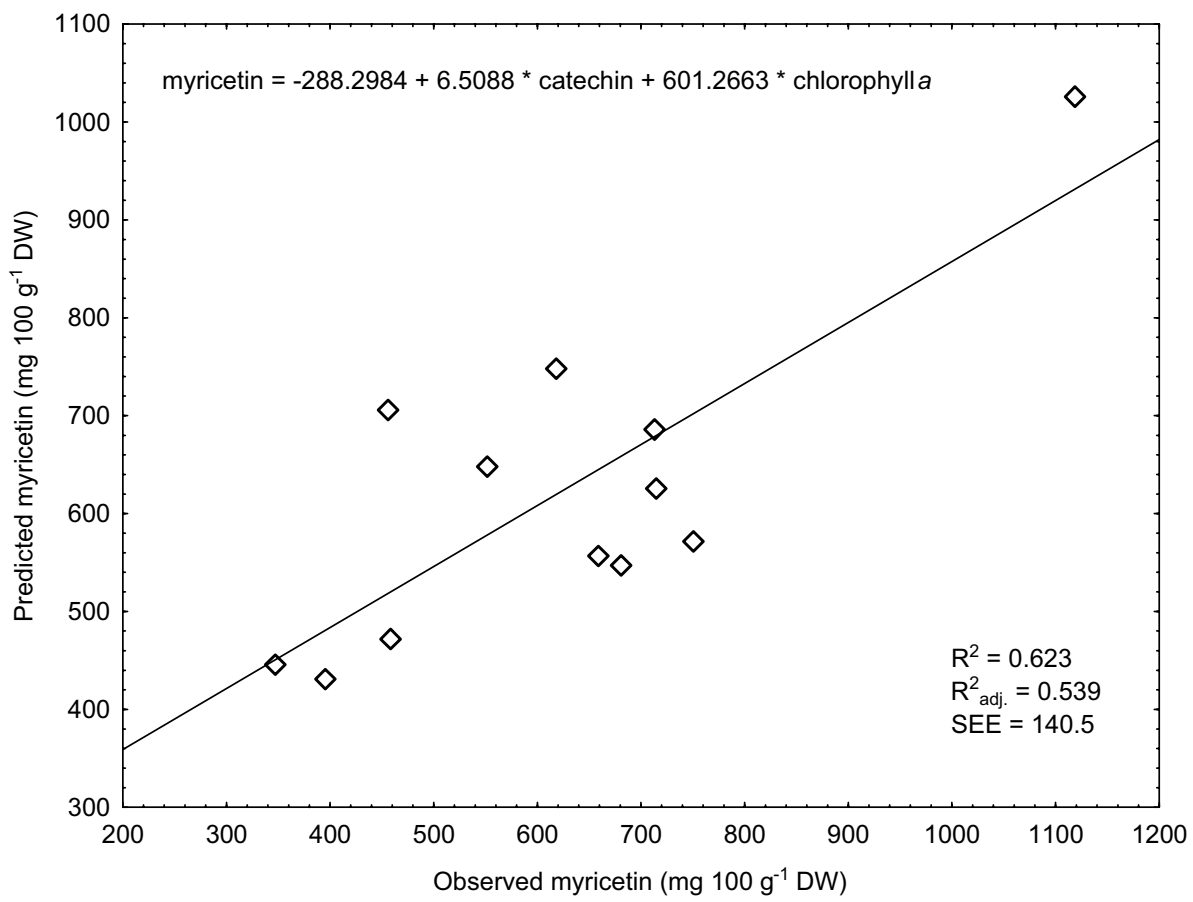

analyses. According to the literature, M. communis leaves contain higher amounts of phenolic compounds compared to flowers, stems or even fruits (Amensour et al. 2009; Aidi Wannes et al. 2010; Aleksic and Knezevic 2014). Among the polyphenols contained in $M$. communis, previous authors have pointed to a significantly higher proportion of flavonoids compared to phenolic acids, which occur in small amounts (Romani et al. 1999; Aleksic and Knezevic 2014; Asgarpanah and Ariamanesh 2015). Our results are consistent with those findings.

Studies of the content of secondary metabolites concerned plant material obtained from natural sites or pot plants, e.g., Saitama Greenery Promotion Center of Kawaguchi City In Japan (Yoshimura et al. 2008), the Greek island Zakynthos (Gardeli et al. 2008), southern Tuscany (Romani et al. 2004), northeastern Tunisia-Nabeul (Aidi Wannes et al. 2010), Zaranjan in the district of Fasa (Taheri et al. 2013) and others (Mansouri et al. 2001; Hayder et al. 2004; Tattini et al. 2006; Amensour et al. 2009; Nassar et al. 2010; Agati et al. 2011; Kumar et al. 2011; Pereira et al. 2012; Tumen et al. 2012; Goncalves et al. 2013; Bouaziz et al. 2015; Babou et al. 2016; Feuillolay et al. 2016). In a study on Myrtus nivelli, Batt \& Trap (Touaibia and Chaouch 2015), the authors found that methanol extracts from in vivo sites were richer in polyphenol content $(348 \mu \mathrm{g}$ eq/mg DW total polyphenol content and $152 \mu \mathrm{g}$ eq/mg DW total flavonoid content) than in vitro culture extracts (respectively 73 and 91). But in those research only one standard was used for polyphenols (gallic acid) and flavonoids (quercetin) total content. Comparing to our studies we used 31 standards for identification phenolic compounds. However, other studies showed that M. communis leaves are richer in antioxidant phenolic compounds at the earlier developmental stage (Babou et al. 2016). In this context, in vitro cultures are a good source of plant material for the production of secondary metabolites. Our research, making use of the RP-HPLC analysis, confirmed the presence of small amounts of phenolic acids and a higher content of flavonoids in $M$. communis extracts. The standards used allowed the identification of gallic, protocatechuic and $p$-hydroxybenzoic acids and catechins and its derivatives, epigallocatechin and epigallocatechin gallate, as well as a particularly important myricetin, which was the most highly abundant of the remaining phenolic compounds in $M$. communis extracts from in vitro cultures. The raw material obtained from in vitro cultures has an additional advantage, because it can be produced at high yields throughout the year, regardless of the growing season. In addition, it will not be contaminated, as sometimes happens in field crops and natural sites (Pierik 1987; Scarpa et al. 2000); moreover, it is homogeneous, with the optimised and desired composition of phenolic antioxidants (Babou et al. 2016). Studies carried out so far on plant material derived from field conditions have shown that polyphenol concentrations and their antioxidant effect is even affected by the season of the year (Gardeli et al. 2008). Further investigations will be necessary to compare samples multiplied in vitro with plant material collected in vivo, due to the high variability of external conditions and their influence on plant material.

In vitro culture is a stressful environment for plants, especially because light determines the direction of 
morphogenesis. If the gas exchange is provided light intensity affects photosynthetic capacity. Too high irradiation can destroy the photosynthetic apparatus and photopigment synthesis (Singh and Patel 2014; Silva et al. 2017). Too low irradiation makes photosynthesis not efficient (Silva et al. 2017). Also for $M$. communis light intensity affects chlorophyll content (Ruffoni et al. 2010). The low irradiation is sufficient for plant morphogenesis in in vitro conditions by providing sucrose in medium (Begna et al. 2002). In addition, the spectral composition of light, such as the ratio of blue and red LED, is important not only for morphogenesis but also for the production of phenolic compound contents (Li and Kubota 2009; Ki-ho and Myung-Min 2013) and secondary metabolism (Silva et al. 2017). For example, $M$. communis has been reported to synthesise a wide array of phenylpropanoids in response to high-light stress (Agati et al. 2011). In our experiment the influence of light quality was investigated and some interesting results were obtained. LED light affected phenolic contents, resulting in an increased content of flavonoids under $100 \% \mathrm{R}$ diodes. The addition of $\mathrm{B}$ light in the spectrum reduced their content, and the use of $100 \%$ B light caused the lowest phenolic contents in the extracts. Li and Kubota (2009) obtained similar light effects on phenolics in leaf lettuce in a greenhouse-red LED addition resulted in increased phenolic content. Furthermore, total flavonoid content was increased under red LED in Rehmannia glutinosa in vitro cultures (Manivannan et al. 2015). The greater content of total phenolic acids under LEDs was also found in Melissa oficinallis cultivated in growing rooms (Frąszczak et al. 2015). The mechanism responsible for this phenomenon is still unknown, but Qamaruddin and Tillberg (1989) concluded that increased phenol contents under red light stimulation could be associated with an increase of cytokinin levels also due to red light.

For this reason, growth regulator levels in media and their appropriate proportions are also important and have an influence on plant development and productivity (Baque et al. 2010). When added to the medium, they can have both a stimulating and inhibiting effect on plant growth (Parzymies and Dąbski 2012). In our research, medium BA content had an effect on phenolic levels in M. communis extracts, as the highest concentration of the cytokinin resulted in the highest level of polyphenols. The lowest content of myricetin was recorded in the application of $2.5 \mu \mathrm{M}$ BA to the medium. Baque et al. (2010) obtained an increased proportion of secondary metabolites, including phenolics and flavonoids in cultures in vitro, as a result of auxin and cytokinin combination in Morinda citrifolia.

The use of appropriate growth regulator proportions has a fundamental influence on biometric parameters of plants cultivated in vitro. The application of BA and NAA in $M$. communis caused a higher shoot multiplication rate (Nobre
1997; Scarpa et al. 2000), while in vitro experiments of the rooting stage by Mascarello et al. (2009) showed that the addition of a small amount of cytokinin caused better rooting of cultured material and a higher chlorophyll level. In the case of Cassia angustifolia, enriching the regulator free MS medium with cytokinins concentration not higher than 5.0 $\mu \mathrm{M}$ caused the induction and increased shoot multiplication rate (Siddique et al. 2015). Nobre et al. (2000) reported that BA concentration in the medium is the most important factor responsible for shoot multiplication of Viburnum tinus. Our study demonstrated that the increasing BA content (1-5 $\mu \mathrm{M})$ in media did not affect $M$. communis multiplication rate, but it inhibited shoot elongation and stimulated leaf formation; shoots in the medium with highest BA concentration $(5 \mu \mathrm{M})$ had the most leaves. The lowest FW content was observed on $2.5 \mu \mathrm{M}$ media, while the largest dry matter content was on the medium with the highest BA content. The highest level of photosynthetic pigments was also observed in media with intermediate BA content $(2.5 \mu \mathrm{M})$. However, the effect of the second factor-light—changed plant responses in some instances. The study conducted by Kozak (2011) showed that the presence of $5 \mu \mathrm{M}$ BA under blue and red light resulted in shoot elongation of Gardenia jasminoides, and a further increase in cytokinin concentration caused elongation inhibition.

Light provides the possibility to manipulate growth conditions in in vitro culture. Light emitting diode systems seem to be very promising for the plant propagation industrywhere light affects growth and development of the plant at each stage. It influences morphogenesis, differentiation of plant cell, tissue and organ cultures ( $\mathrm{Li}$ et al. 2010; Gupta and Jatothu 2013) as well as the proliferation rate (Sæbø et al. 1995), which could be essential for the production of secondary metabolites. It was shown in M. communis that light intensity during the rooting phase in vitro could modulate biomass production (Ruffoni et al. 2010). In our study, light quality affected multiplication rate and chlorophyll content. Analysing both the effect of light and medium composition, multiplication rate was greatest under R LED light and highest BA content compared to other combinations. However, higher concentrations of cytokinin inhibited the multiplication rate when B LED light was used. Moreover, low levels of BA in combination with R LED light provided longer multiplied shoots. Blue LED light with high BA content stimulated the growth of a greater number of M. communis leaves. Manivannan et al. (2015) showed that the effect of red light stimulated endogenous gibberellins involved in mitosis and cell proliferation. Meanwhile, blue light improved leaf characteristics, such as leaf number, and modifications in spectrum composition and light quality are easily perceived by leaf photoreceptors, which affect their morphogenesis. Our study showed a lower concentration of photosynthetic pigments in M. communis cultured 
under LED light compared to the fluorescent lamp light. The lowest content of these compounds was recorded under blue LED light. The highest concentration was observed for plants grown on the medium supplemented with $2.5 \mu \mathrm{M}$ BA. Numerous studies in other plant species have been conducted to investigate the effects of LED lighting in in vitro cultures (Gupta and Jatothu 2013). Some of the results were consistent with our study: red light increased multiplication rate (Mengxi et al. 2011) and shoot elongation (Hahn et al. 2000; Heo et al. 2002; Kim et al. 2004; Poudel et al. 2008), whereas monochromatic blue caused a greater number of leaves (Macedo et al. 2011; Manivannan et al. 2015). However, in contrast to our results, photosynthetic pigment content was elevated in some studies after LED light application, especially monochromatic blue (Jao et al. 2005; Kurilčik et al. 2008; Poudel et al. 2008; Manivannan et al. 2015). The work of Lin et al. (2013) showed that light quality treatments did not significantly affect chlorophyll or carotenoid contents. There are also reports in the literature on changes in FW and DW in plant cultures in vitro under various light conditions. Our study demonstrated that LED light with the addition of blue spectrum (B and RB) increased the FW compared to fluorescent lamps, but did not affect the DW. The results obtained by Kim et al. (2004), Jeong et al. (2006), Moon et al. (2006), Li et al. (2010, 2013), Lin et al. (2013) and Manivannan et al. (2015) confirmed the effect of mixed LEDs on FW increase and also showed a similar tendency for DW. Despite the effect of B and R LED on the growth and development of plants through photoreceptor stimulation, the results of many studies are inconsistent. It is difficult to understand how plants respond to changes in light quality because studies compare only specific ratios in many different species, and their responses are often contradictory (Ki-ho and Myung-Min 2013; Wojciechowska et al. 2016). However, the best results were often obtained with a mixture of red and blue light, where blue LED influenced chlorophyll formation and chloroplast development rather than having a direct effect on biomass accumulation and elongation growth, as exerted by red light (Shin et al. 2008; Li and Kubota 2009; Ki-ho and Myung-Min 2013).

\section{Conclusions}

Light is critical for the in vitro cultivation of $M$. communis L., since it affects the growth, morphogenesis and production of phytochemical compounds. Our research has shown a stimulating effect of red light on multiplication rate, shoot height and the highest increase in antioxidant polyphenol concentrations. Therefore, the use of $5 \mu \mathrm{M}$ BA in the medium produced better results in terms of increasing multiplication rate, leaf number, DW and polyphenol concentrations compared to the lower content of this cytokinin.
Under the controlled in vitro conditions, conscious manipulation of light quality, coupled with the benefits of LED technology, will contribute to the economic enhanced biomass production with high secondary metabolite contents. Furthermore, it will ensure obtaining homogeneous material in a relatively short period of time without the need to cultivate the plant to the fructification stage or collecting organic matter from fully developed plants from field conditions.

Acknowledgements This work was supported by the Polish Ministry of Science and Higher Education (DS 3500).

Author contributions The following declarations about authors contributions to the research have been made: concept of the study: BP; LED light system-design and settings: MŻ; laboratory research: MC, AS; statistical analyses: MC, AK, BP; writing of the manuscript $\mathrm{MC}, \mathrm{BP}, \mathrm{AK}, \mathrm{AS}$.

\section{Compliance with ethical standards}

Conflict of interest The authors declare no conflict of interests.

Open Access This article is distributed under the terms of the Creative Commons Attribution 4.0 International License (http://creativecommons.org/licenses/by/4.0/), which permits unrestricted use, distribution, and reproduction in any medium, provided you give appropriate credit to the original author(s) and the source, provide a link to the Creative Commons license, and indicate if changes were made.

\section{References}

Agati G, Cerovic ZG, Pinelli P, Tattini M (2011) Light-induced accumulation of ortho-dihydroxylated flavonoids as nondestructively monitored by chlorophyll fluorescence excitation techniques. Environ Exp Bot 73:3-9. https://doi.org/10.1016/j. envexpbot.2010.10.002

Ahmadvand H, Bagheri S (2011) Inhibitory effects of myrtle (Myrtus communis L) leaves extract on LDL oxidation in vitro. Clin Biochem 44:341-342. https://doi.org/10.1016/j. clinbiochem.2011.08.850

Aidi Wannes W, Mhamdi B, Sriti J et al (2010) Antioxidant activities of the essential oils and methanol extracts from myrtle (Myrtus communis var. italica L.) leaf, stem and flower. Food Chem Toxicol 48:1362-1370. https://doi.org/10.1016/j.fct.2010.03.002

Aka Kaçar Y, Şimşek Ö, Biçen B, Dal B (2017) In vitro rooting of micropropagated shoots from Myrtus communis Linn: influence of activated charcoal and indole-3-butyric acid (IBA). Acta Hortic 531-536. https://doi.org/10.17660/ActaHortic.2017.1155.78

Alamanni MC, Cossu M (2004) Radical scavenging activity and antioxidant activity of liquors of myrtle (Myrtus communis L.) berries and leaves. Ital J Food Sci 16:197-208

Aleksic V, Knezevic P (2014) Antimicrobial and antioxidative activity of extracts and essential oils of Myrtus communis L. Microbiol Res 169:240-254. https://doi.org/10.1016/j.micres.2013.10.003

Alipour G, Dashti S, Hosseinzadeh H (2014) Review of pharmacological effects of Myrtus communis L. and its active constituents. Phytother Res 28:1125-1136

Alvarenga ICA, Pacheco FV, Silva ST et al (2015) In vitro culture of Achillea millefolium L.: quality and intensity of light on 
growth and production of volatiles. Plant Cell Tissue Organ Cult 122:299-308. https://doi.org/10.1007/s11240-015-0766-7

Amensour M, Sendra E, Abrini J et al (2009) Total phenolic content and antioxidant activity of myrtle (Myrtus communis) extracts. Nat Prod Commun 4:819-824

Amessis-Ouchemoukh N, Madani K, Fale PL V et al (2014) Antioxidant capacity and phenolic contents of some Mediterranean medicinal plants and their potential role in the inhibition of cyclooxygenase- 1 and acetylcholinesterase activities. Ind Crop Prod 53:6-15. https://doi.org/10.1016/j.indcrop.2013.12.008

Asgarpanah J, Ariamanesh A (2015) Phytochemistry and pharmacological properties of Myrtus communis L. Indian J Tradit Knowl $1: 82-87$

Babou L, Hadidi L, Grosso C et al (2016) Study of phenolic composition and antioxidant activity of myrtle leaves and fruits as a function of maturation. Eur Food Res Technol 242:1447-1457. https:// doi.org/10.1007/s00217-016-2645-9

Badra B, Saoudi A, Akila A (2016) Essential oil chemical composition of myrtle growing in Northeastern Algeria and estimation of its antibacterial effectiveness. Am J Biochem Biotechnol 12:110 121. https://doi.org/10.3844/ajbbsp.2016.110.121

Bajalan I, Ghasemi Pirbalouti A (2014) Variation in antibacterial activity and chemical compositions of essential oil from different populations of myrtle. Ind Crop Prod 61:303-307. https://doi. org/10.1016/j.indcrop.2014.07.023

Balasundram N, Sundram K, Samman S (2006) Phenolic compounds in plants and agri-industrial by-products: antioxidant activity, occurrence, and potential uses. Food Chem 99:191-203. https://doi. org/10.1016/j.foodchem.2005.07.042

Baque MA, Hahn E-J, Paek K-Y (2010) Growth, secondary metabolite production and antioxidant enzyme response of Morinda citrifolia adventitious root as affected by auxin and cytokinin. Plant Biotechnol Rep 4:109-116. https://doi.org/10.1007/ s11816-009-0121-8

Begna SH, Dwyer LM, Cloutier D et al (2002) Decoupling of light intensity effects on the growth and development of $\mathrm{C} 3$ and $\mathrm{C} 4$ weed species through sucrose supplementation. J Exp Bot 53:1935-1940. https://doi.org/10.1093/jxb/erf043

Benkhayal FA, Musbah E, Ramesh S, Dhayabaran D (2009) Biochemical studies on the effect of phenolic compounds extracted from Myrtus communis in diabetic rats. Tamilandu J Vet Anim Sci 5:87-93

Bouaziz A, Khennouf S, Zarga MA et al (2015) Phytochemical analysis, hypotensive effect and antioxidant properties of Myrtus communis L. growing in Algeria. Asian Pac J Trop Biomed 5:19-28. https://doi.org/10.1016/S2221-1691(15)30165-9

Canhoto JM, Lopes ML, Cruz GS (1999) Somatic embryogenesis and plant regeneration in myrtle (Myrtaceae). Plant Cell Tissue Organ Cult 57:13-21

Ellnain-Wojtaszek M, Zgórka G (1999) High-performance liquid chromatography and thin-layer chromatography of phenolic acids from Ginkgo biloba L. leaves collected within vegetative period. J Liq Chromatogr Relat Technol 22:1457-1471. https:// doi.org/10.1081/JLC-100101744

Feuillolay C, Pecastaings S, Gac C, Le et al (2016) A Myrtus communis extract enriched in myrtucummulones and ursolic acid reduces resistance of Propionibacterium acnes biofilms to antibiotics used in acne vulgaris. Phytomedicine 23:307-315. https://doi. org/10.1016/j.phymed.2015.11.016

Frąszczak B, Gąsecka M, Golcz A, Zawirska-Wojtasiak R (2015) The chemical composition of lemon balm and basil plants grown under different light conditions. Acta Sci Pol-Hortoru 14:93-104

Frohne D, Classen B (2006) Heilpflanzenlexikon - Ein Leitfaden auf wissenschaftlicher Grundlage. Wissenschaftliche Verlagsgesellschaft, Stuttgart, pp 346-347
Gardeli C, Vassiliki P, Athanasios M et al (2008) Essential oil composition of Pistacia lentiscus L. and Myrtus communis L.: evaluation of antioxidant capacity of methanolic extracts. Food Chem 107:1120-1130. https://doi.org/10.1016/j.foodchem.2007.09.036

Goncalves S, Gomes D, Costa P, Romano A (2013) The phenolic content and antioxidant activity of infusions from Mediterranean medicinal plants. Ind Crop Prod 43:465-471. https://doi. org/10.1016/j.indcrop.2012.07.066

Gupta SD, Jatothu B (2013) Fundamentals and applications of lightemitting diodes (LEDs) in in vitro plant growth and morphogenesis. Plant Biotechnol Rep 7:211-220. https://doi.org/10.1007/ s11816-013-0277-0

Hahn E, Kozai T, Paek K (2000) Blue and red light-emitting diodes with or without sucrose and ventilation affect in vitro growth of Rehmannia glutinosa plantlets. J Plant Biol 43:247-250. https:// doi.org/10.1007/BF03030425

Harborne JB (1998) Phytochemical methods. A guide to modern techniques of plant analysis. Chapman \& Hall, London, pp 63-64

Haron NW, Moore DM, Harborne JB (1992) Distribution and taxonomic significance flavonoids in the genus Eugenia (Myrtaceae). Biochem Syst Ecol 20:266-268. https://doi. org/10.1016/0305-1978(89)90085-9

Hayder N, Abdelwahed A, Kilani S et al (2004) Anti-genotoxic and free-radical scavenging activities of extracts from (Tunisian) $M y r-$ tus communis. Mutat Res 564:89-95. https://doi.org/10.1016/j. mrgentox.2004.08.001

Hayder N, Bouhlel I, Skandrani I et al (2008) In vitro antioxidant and antigenotoxic potentials of myricetin-3-o-galactoside and myricetin-3-o-rhamnoside from Myrtus communis: modulation of expression of genes involved in cell defence system using cDNA microarray. Toxicol In Vitro 22:567-581. https://doi.org/10.1016/j. tiv.2007.11.015

Heo J, Lee C, Chakrabarty D, Paek K (2002) Growth responses of marigold and salvia bedding plants as affected by monochromic or mixture radiation provided by a light-emitting diode (LED). Plant Growth Regul 38:225-230. https://doi.org/10.102 3/A:1021523832488

Hoppe HA (1975) Drogen kunde. Walter de Gruyter, Berlin, p. 740

Jao RC, Lai CC, Fang W, Chang SF (2005) Effects of red light on the growth of Zantedeschia plantlets in vitro and tuber formation using light-emitting diodes. HortScience 40:436-438

Jędrzejko K, Klama H, Żarnowiec J (1997) Medicinal plants (Zarys wiedzy o roślinach leczniczych). Śląska Akademia Medyczna w Katowicach, Katowice, pp 229-230

Jeong WH, Kong SS, Seon KK, Kee YP (2006) Light quality affects in vitro growth of grape 'Teleki 5BB'. J Plant Biol 49:276-280. https://doi.org/10.1007/BF03031155

Kalachanis D, Psaras GK (2005) Structure and development of the secretory cavities of Myrtus communis leaves. Biol Plant 49:105110. https://doi.org/10.1007/s00000-005-5110-2

Ki-ho S, Myung-Min O (2013) Leaf shape, growth, and antioxidant phenolic compounds of two lettuce cultivars grown under various combinations of blue and red light-emitting diodes. HortScience 48:988-995

Kim SJ, Hahn EJ, Heo JW, Paek KY (2004) Effects of LEDs on net photosynthetic rate, growth and leaf stomata of chrysanthemum plantlets in vitro. Sci Hortic 101:143-151. https://doi. org/10.1016/j.scienta.2003.10.003

Kozak D (2011) The influence of light quality and ba on in vitro growth and development of Gardenia jasminoides Ellis. Acta Sci PolHortoru 10:65-73

Kumar RM, Phaneedra P, Bodhanapu S et al (2011) Antioxidant and hepatoprotective activity of the aqueous extract of Myrtus communis (Myrtle) Linn. leaves. Pharmacologyonline 1:1083-1090 
Kurilčik A, Miklušytė-Čanova R, Dapkūnienė S et al (2008) In vitro culture of Chrysanthemum plantlets using light-emitting diodes. Cent Eur J Biol 3:161-167. https://doi.org/10.2478/ s11535-008-0006-9

Lattanzio V (2013) Phenolic compounds: introduction. Nat Prod. https://doi.org/10.1007/978-3-642-22144-6_57

Li Q, Kubota C (2009) Effects of supplemental light quality on growth and phytochemicals of baby leaf lettuce. Environ Exp Bot 67:5964. https://doi.org/10.1016/j.envexpbot.2009.06.011

Li H, Xu Z, Tang C (2010) Effect of light-emitting diodes on growth and morphogenesis of upland cotton (Gossypium hirsutum L.) plantlets in vitro. Plant Cell Tissue Organ Cult 103:155-163. https://doi.org/10.1007/s11240-010-9763-Z

Li H, Tang C, Xu Z (2013) The effects of different light qualities on rapeseed (Brassica napus L.) plantlet growth and morphogenesis in vitro. Sci Hortic 150:117-124. https://doi.org/10.1016/j. scienta.2012.10.009

Lian ML, Murthy HN, Paek KY (2002) Effects of light emitting diodes (LEDs) on the in vitro induction and growth of bulblets of $\mathrm{Lil}$ ium oriental hybrid 'Pesaro'. Sci Hortic 94:365-370. https://doi. org/10.1016/S0304-4238(01)00385-5

Lichtenthaler HK, Buschmann C (2001) Chlorophylls and carotenoids: measurement and characterization by UV-VIS spectroscopy. Curr Protoc Food Anal Chem. https://doi.org/10.1002/0471709085. $\operatorname{ch} 21$

Lim TK (2012) Myrtus communis. In: Edible medicinal and non medicinal plants. Volume 3, Fruits. Springer, Dordrecht, pp 642654. https://doi.org/10.1007/978-94-007-2534-8

Lin Y, Li J, Li B et al (2011) Effects of light quality on growth and development of protocorm-like bodies of Dendrobium officinale in vitro. Plant Cell Tiss Organ Cult 105:329-335. https://doi. org/10.1007/s11240-010-9871-9

Lin KH, Huang MY, Huang WD et al (2013) The effects of red, blue, and white light-emitting diodes on the growth, development, and edible quality of hydroponically grown lettuce (Lactuca sativa L. var. capitata). Sci Hortic 150:86-91. https://doi.org/10.1016/j. scienta.2012.10.002

Macedo AF, Leal-Costa MV, Tavares ES et al (2011) The effect of light quality on leaf production and development of in vitro-cultured plants of Alternanthera brasiliana Kuntze. Environ Exp Bot 70:43-50. https://doi.org/10.1016/j.envexpbot.2010.05.012

Magherini R (1988) Le piante medicinali e aromatiche ieri e oggi. Possibilitá di coltivazione delle piante medicinali e aromatiche. L'Italia Agricola 3:17-22

Manivannan A, Soundararajan P, Halimah N, Ko CH (2015) Blue LED light enhances growth, phytochemical contents, and antioxidant enzyme activities of Rehmannia glutinosa cultured in vitro. Hort Environ Biotechnol 56:105-113. https://doi.org/10.1007/ s13580-015-0114-1

Mansouri S, Foroumadi A, Ghaneie T, Najar AG (2001) Antibacterial activity of the crude extracts and fractionated constituents of Myrtus communis. Pharm Biol 39:399-401

Mascarello C, Melis R, Mantovani E, Ruffoni B (2009) Evaluation of the role of light intensity and hormonal treatments during in vitro rooting of Myrtus micropropagated plantlets related to acclimatization performances. Acta Hortic 812:379-386

Massa GD, Kim H, Wheeler RM, Mitchell CA (2008) Plant productivity in response to LED lighting. HortScience 43:1951-1956

Mengxi L, Zhigang X, Yang Y, Yijie F (2011) Effects of different spectral lights on Oncidium PLBs induction, proliferation, and plant regeneration. Plant Cell Tissue Organ Cult 106:1-10. https://doi. org/10.1007/s11240-010-9887-1

Miean KH, Mohamed S (2001) Flavonoid (myricetin, qercetin, kaempferol, luteolin, and apigenin) content of edible tropical plants. J Agric Food Chem 49:3106-3112
Moon HK, Park S-Y, Kim YW, Kim CS (2006) Growth of Tsuru-rindo (Tripterospermum japonicum) cultured in vitro under various sources of light-emitting diode (LED) irradiation. J Plant Biol 49:174-179. https://doi.org/10.1007/BF03031014

Murashige T, Skoog F (1962) A revised medium for rapid growth and bio assays with tobacco tissue cultures. Physiol Plantarum 15:473-497

Nassar MI, Aboutabl EA, Ahmed RF et al (2010) Secondary metabolites and bioactivities of Myrtus communis. Pharmacogn Res 2:325-329. https://doi.org/10.4103/0974-8490.75449

Nhut DT, Takamura T, Watanabe H et al (2003) Responses of strawberry plantlets cultured in vitro under superbright red and blue light-emitting diodes (LEDs). Plant Cell Tissue Organ Cult 73:43-52. https://doi.org/10.1023/A:1022638508007

Nobre J (1997) Micropropagation of Myrtus communis L. (Mediterranean myrtle). Biotechnol Agric For 39:127-134

Nobre J, Santos C, Romano A (2000) Micropropagation of the Mediterranean species Viburnum tinus. Plant Cell Tissue Organ Cult 60:75-78

Parra R, Amo-Marco JB (1998) Factors affecting in vitro shoot proliferation of Myrtus communis L.: a comparison of adult and seedling material. In Vitro Cell Dev Biol-Plant 34:104-107

Parra R, Pastor MT, Pérez-Payá E, Amo-Marco JB (2001) Effect of in vitro shoot multiplication and somatic embryogenesis on 5-methylcytosine content in DNA of Myrtus communis L. Plant Growth Regul 33:131-136. https://doi.org/10.102 3/A:1017571911028

Parzymies M, Dąbski M (2012) The effect of cytokinin types and their concentration on in vitro multiplication of Clematis viticella (L.) and Clematis integrifolia 'Petit Faucon'. Acta Sci PolHortoru 11:81-91

Pereira P, Cebola M-J, Bernardo-Gil MG (2012) Comparison of antioxidant activity in extracts of Myrtus communis L. obtained by SFE vs. solvent extraction. J Environ Sci Eng A 1:115-120

Pierik R (1987) In vitro culture of higher plants. Martinus Nijhoff Publishers, The Hague

Poudel PR, Kataoka I, Mochioka R (2008) Effect of red- and blue-light-emitting diodes on growth and morphogenesis of grapes. Plant Cell Tissue Organ Cult 92:147-153. https://doi. org/10.1007/s11240-007-9317-1

Qamaruddin M, Tillberg E (1989) Rapid effects of red light on the isopentenyladenosine content in Scots pine seeds. Plant Physiol 91:5-8

Rezaee A, Kamali K (2014) A new commercial protocol for micropropagation of Myrtus tree. Adv Biores 5:73-79. https://doi. org/10.15515/abr.0976-4585.5.4.7379

Romani A, Pinelli P, Mulinacci N et al (1999) Identification and quantitation of polyphenols in leaves of Myrtus communis L. Chromatographia 49:17-20

Romani A, Coinu R, Carta S et al (2004) Evaluation of antioxidant effect of different extracts of Myrtus communis L. Free Radic Res 38:97-103. https://doi.org/10.1080/10715760310001625 609

Romani A, Campo M, Pinelli P (2012) HPLC/DAD/ESI-MS analyses and anti-radical activity of hydrolyzable tannins from different vegetal species. Food Chem 130:214-221. https://doi. org/10.1016/j.foodchem.2011.07.009

Ruffoni B, Mascarello C, Savona M (2010) In vitro propagation of ornamental myrtus (Myrtus communis). Methods Mol Biol 589:257-269

Sæbø A, Krekling T, Appelgren M (1995) Light quality affects photosynthesis and leaf anatomy of birch plantlets in vitro. Plant Cell Tissue Organ Cult 41:177-185. https://doi.org/10.1007/ BF00051588 
Şan B, Karakurt Y, Dönmez F (2015) Effects of thidiazuron and activated charcoal on in vitro shoot proliferation and rooting of Myrtle (Myrtus communis L.). J Agric Sci 21:177-183

Scarpa GM, Milia M, Satta M (2000) The influence of growth regulators on shoot proliferation and rooting of in vitro propagated myrtle. Plant Cell Tissue Organ Cult 62:175-179

Shin KS, Murthy HN, Heo JW et al (2008) The effect of light quality on the growth and development of in vitro cultured Doritaenopsis plants. Acta Physiol Plant 339-343. https://doi.org/10.1007/ s11738-007-0128-0

Siddique I, Abdullah N, Bukhari W et al (2015) Influence of plant growth regulators on in vitro shoot multiplication and plantlet formation in Cassia angustifolia Vahl. Braz Arch Biol Technol 58:686-691

Silva ST, Bertolucci SKV, da Cunha SHB et al (2017) Effect of light and natural ventilation systems on the growth parameters and carvacrol content in the in vitro cultures of Plectranthus amboinicus (Lour.) Spreng. Plant Cell Tissue Organ Cult 129:501-510. https://doi.org/10.1007/s11240-017-1195-6

Singh P, Patel RM (2014) Factors influencing in vitro growth and shoot multiplication of pomegranate. Bioscan 9:1031-1035

Sumbul S, Ahmad MA, Asif M, Akhtar M (2011) Myrtus communis Linn.- - review. Indian J Nat Prod Resour 2:395-402

Taheri A, Seyfan A, Jalalinezhad S, Nasery F (2013) Antibacterial effect of Myrtus communis hydro-alcoholic extract on pathogenic bacteria. Zahedan J Res Med Sci 15:19-24

Tattini M, Remorini D, Pinelli P et al (2006) Morpho-anatomical, physiological and biochemical adjustments in response to root zone salinity stress and high solar radiation in two Mediterranean evergreen shrubs, Myrtus communis and Pistacia lentiscus. New Phytol 170:779-794

Touaibia M, Chaouch FZ (2015) Propriétés antioxydantes et antimicrobiennes des extraits de Myrtus nivellei Batt et Trab. obtenus in situ et in vitro. Phytothérapie. https://doi.org/10.1007/ s10298-015-1011-6

Tumen I, Senol FS, Orhan IE (2012) Inhibitory potential of the leaves and berries of Myrtus communis L. (myrtle) against enzymes linked to neurodegenerative diseases and their antioxidant actions. Int J Food Sci Nutr 63:387-392. https://doi.org/10.3109/096374 86.2011.629178

Vitrac X, Krisa S, Decendit A et al (2004) Grapevine polyphenols and their biological effects. In: Ramawat KG (ed) Biotechnology of medicinal plants-vitalizer and therapeutic. Science Publishers, Enfield, New Hampshire, pp 33-75

Wojciechowska R, Kurpaska S, Malinowski M et al (2016) Effect of supplemental led lighting on growth and quality of Valerianella locusta $\mathrm{L}$. and economic aspects of cultivation in autumn cycle. Acta Sci Pol-Hortoru 15:233-244

Yildirim F, San B, Yildirim AN et al (2015) Mineral composition of leaves and fruit in some myrtle (Myrtus communis L.) genotypes. Erwerbs-Obstbau 57:149-152. https://doi.org/10.1007/ s10341-015-0243-9

Yoshimura M, Amakura Y, Tokohura M, Yoshida T (2008) Polyphenolic compounds isolated from the leaves of Myrtus communis. $\mathrm{J}$ Nat Med 62:366-368. https://doi.org/10.1007/s11418-008-0251-2 